The Astronomical Journal, 122:3419-3435, 2001 December

(C) 2001. The American Astronomical Society. All rights reserved. Printed in U.S.A.

\title{
A SURVEY OF PROPER-MOTION STARS. XIV. SPECTROSCOPIC BINARIES AMONG METAL-POOR FIELD BLUE STRAGGLERS ${ }^{1}$
}

\author{
BRUCE W. CARNEY \\ Department of Physics and Astronomy, University of North Carolina, Chapel Hill, NC 27599-3255; bruce@physics.unc.edu \\ DAVID W. LATHAM \\ Harvard-Smithsonian Center for Astrophysics, 60 Garden Street, Cambridge, MA 02138; dlatham@cfa.harvard.edu \\ JOHN B. LAIRD \\ Department of Physics and Astronomy, Bowling Green State University, Bowling Green, OH 43403; laird@tycho.bgsu.edu \\ CATHERINe E. GRANT ${ }^{2}$ \\ Department of Astronomy, Harvard University, Cambridge, MA 02138; cgrant@space.mit.edu \\ AND \\ JoN A. MorSE \\ Center for Astrophysics and Space Astronomy, Department of Astrophysical and Planetary Sciences, University of Colorado, Campus Box 389, \\ Boulder, CO 80309; morsey@casa.colorado.edu \\ Received 1999 May 4; accepted 2001 September 5
}

\begin{abstract}
We summarize the results from a program of monitoring the radial velocities of 10 metal-poor, highvelocity field stars whose colors are 0.01 to $0.13 \mathrm{mag}$ bluer than main-sequence turnoffs of comparablemetallicity globular clusters. Two of the candidate halo blue stragglers (BD +72 94 and BD +40 1166) show no signs of velocity variability, one (HD 84937) shows only weak signs of variability, one (BD + 25 1981) appears to be a very long-period binary, and six (BD - 12 2669, HD 97916, HD 106516, BD + 51 1817, G66-30, and G202-65) are single-lined spectroscopic binaries, with periods ranging from 167 to 844 days. Velocity coverage for the four candidates without orbital solutions ranges from 15.9 to 19.0 years. The orbital eccentricities are all low, $e<0.30$ and $\langle e\rangle=0.11$. Five of the six binary orbits have very low eccentricities, with $\langle e\rangle=0.07$. We have reanalyzed the velocity data from Preston \& Sneden and have derived orbital solutions similar to theirs for 10 of the spectroscopic binaries among their "blue metal-poor" stars with $[\mathrm{Fe} / \mathrm{H}] \leq-0.6$. We confirm their conclusion that the binary frequency is high; we find $47 \pm 10 \%$ if we include only the definite binaries with $[\mathrm{Fe} / \mathrm{H}] \leq-0.6$. Our orbital solutions for the seven binaries with periods longer than 20 days all have low eccentricities, with $e \leq 0.26$ and $\langle e\rangle=0.11$. These orbital characteristics are very similar to the $\mathrm{Ba}$ II, $\mathrm{CH}$, subgiant $\mathrm{CH}$, and dwarf carbon stars, suggesting that mass transfer has been involved in their formation. Of the five binary stars in our program with published abundances of lithium, all have been found to be deficient (and one in beryllium as well). In contrast, two of the three apparently single stars have published lithium abundances and show no deficiency. The mass functions for the six binaries in our program and seven similar systems studied by Preston \& Sneden are consistent with their unseen companions all being white dwarfs with $M \approx 0.55 M_{\odot}$ and random orbital inclinations. Taking all of our observations and those of others together, we argue that the results are consistent with all field blue stragglers being binary systems with long periods and low eccentricities, the primary stars being deficient in lithium and the secondary stars being normal-mass white dwarfs. All these properties are suggestive of a blue-straggler formation model that involves mass transfer. For six of the 13 stars in the two programs for which sprocess elemental abundances are available, no signs of enhancement are discernible, suggesting that the donor star was a first-ascent red giant. For the star with the longest orbital period (1307 days), CS 22956-028, s-process abundance enhancements have been reported. This star may be a precursor to the subgiant $\mathrm{CH}$ class, as suggested by Luck \& Bond.
\end{abstract}

Key words: binaries: spectroscopic — blue stragglers — Galaxy: halo

\section{INTRODUCTION}

Sandage (1953) published the first color-magnitude diagram of the globular cluster M3 that was deep enough to reach the main sequence. He called attention to a modest number of stars brighter and bluer than the main-sequence turnoff. These, and similar stars found in both globular clusters and open clusters, are the "blue stragglers."

\footnotetext{
${ }^{1}$ Some of the results presented here used observations made with the Multiple Mirror Telescope, a joint facility of the Smithsonian Institution and the University of Arizona.

${ }^{2}$ Now at the Center for Space Research, Massachusetts Institute of Technology, Cambridge, MA 02139.
}

The research on blue stragglers has been extensive, and excellent reviews are given by Stryker (1993), Livio (1993), Trimble (1993), Bailyn (1995), and Leonard (1996). Why has so much attention been paid to such pathological stars?

If blue stragglers are and always have been single stars, their existence implies that either more than one epoch of star formation has occurred in the clusters in which they are found or that some unknown process has slowed the stellar evolution clock. Is something crucial missing from our understanding of stellar structure and evolution on the main sequence? Are blue stragglers a field of "stellar pathology" to help us learn how stars work? Or is there a more benign explanation? 
Blue stragglers appear to be more massive than the mainsequence turnoff. Shara, Saffer, \& Livio (1997) derived atmospheric parameters for the blue straggler BSS 19 in the globular cluster 47 Tucanae, implying a mass of $1.7 \pm 0.4$ $M_{\odot}$. Indirect mass estimates also support the high masses. The radial distribution of blue stragglers in the dynamically evolved cluster NGC 5053 supports typical masses of $\approx 1.2$ $M_{\odot}($ Nemec \& Cohen 1989), while pulsational analyses of SX Phe variables (hotter blue stragglers inside the instability strip) suggest masses of between 1.2 and $1.4 M_{\odot}$ (Nemec et al. 1995). Recent star formation in M3 and other globular clusters can be ruled out from their lack of gas. Any gas that does accumulate from stellar mass loss will be cleared as a cluster passes periodically through the Galactic disk every hundred million years or so. If stellar evolution theory is at fault, we must understand why, because so much of modern astrophysics rests on it. The choices are that either the blue stragglers are normal and most stars evolve faster than predicted or that the minority blue stragglers are abnormal and evolve too slowly.

In the first case, Willson, Bowen, \& Struck-Marcell (1987) suggested that stars with $M=1-3 M_{\odot}$ could lose sufficient mass through pulsationally driven stellar winds to move down the main sequence. The blue stragglers would somehow have been left behind. This can probably be ruled out from studies of lithium abundances in blue stragglers and normal main-sequence stars. Lithium is "fragile," being destroyed by the ${ }^{7} \mathrm{Li}(p, \alpha)^{4} \mathrm{He}$ reaction at relatively cool temperatures (of order $2 \times 10^{6} \mathrm{~K}$ ). In metal-poor dwarfs hotter than about $5400 \mathrm{~K}$, lithium is found in stellar photospheres (Thorburn 1994 and references therein; also Ryan, Norris, \& Beers 1999). (For cooler temperatures, lithium abundances in main-sequence stars decrease, presumably because of the increasing size of the convection zone and the transport of lithium to layers deep and hot enough for its destruction.) Hotter stars, such as blue stragglers, do not have deep convection zones that could transport the lithium readily to such depths, and so they would be expected to have "normal" lithium abundances. However, lithium is not found in the blue stragglers studied to date (Hobbs \& Mathieu 1991; Pritchet \& Glaspey 1991; Glaspey, Pritchet, \& Stetson 1994; Ryan et al. 2001a).

The second case, that the evolution of blue stragglers away from the main sequence may be delayed, could be explained if the stars were somehow to mix more thoroughly their interiors, bringing fresh hydrogen fuel to their cores (Wheeler 1979a, 1979b; Saio \& Wheeler 1980). The absence of lithium in blue-straggler photospheres could be taken as evidence that such mixing has reached the surface layers, transporting the lithium to the deeper layers where it is destroyed. However, mass transfer or complete merger within a binary system could also provide extra fuel and thereby prolong the main-sequence lifetime and thus explain the absence of lithium (since the new surface layers would presumably have been donated from the deeper and hotter layers of the donor star). The enhanced $s$-process abundances found by Mathys (1991) in two blue stragglers in the old open cluster M67 could be explained by mass transfer from a core helium-burning star. Mass transfer is the more benign explanation, first noted by McCrea (1964), since it would not require revisions to our understanding of single-star evolutionary processes.

The focus on blue stragglers has therefore shifted to the consequences of binary-star evolution in many different situations. As the several reviews cited above have discussed, blue stragglers are common in high-density globular cluster cores, suggesting that binary-star evolution and interactions can lead to collisions, mergers, or harder binaries that are more likely to lead to mass transfer. Lower density clusters also contain significant numbers of blue stragglers (but more centrally concentrated due to the effects of stellar dynamics and mass segregation), suggesting that isolated "primordial" binary systems may also lead to blue stragglers. There are two especially interesting cases of clusters where the blue-straggler frequency is high in the high-density central regions, where dynamical effects presumably accelerate binary formation and evolution, low in the intermediate radial regions, where binary disruption might be the rule, and high again in the distant, low-density outer regions, where binary systems may evolve in isolation (M3: Ferraro et al. 1993; M80: Ferraro, Fusi Pecci, \& Bellazzini 1995).

To improve our understanding of blue stragglers, particularly the binary-star aspects, a wide variety of studies is needed. Globular clusters, as well as some open clusters, offer us a means to probe the consequences of environment on the formation of blue stragglers. However, to study the blue stragglers formed as a consequence of isolated binarystar evolution, we need to sample low-density environments: low-density clusters or field stars. Long-term spectroscopic studies must be undertaken to determine binary fractions and the orbital characteristics of the binaries discovered thereby. And we need detailed chemical composition analyses to better probe aspects of mass transfer in these systems. Field stars offer us an opportunity for such work.

We report here on a long-term program to monitor the radial velocities of 10 candidate field blue stragglers. We present orbital solutions for six of the 10 candidates and employ their orbital characteristics in comparison with "normal" metal-poor field binary systems to argue that mass transfer has occurred. Finally, we offer suggestions for future work.

\section{IDENTIFICATION OF FIELD BLUE STRAGGLERS}

Identifying blue stragglers in the field halo population is not as simple as in globular clusters, but there have been several searches for such stars. These are based primarily on identifying high-gravity stars whose colors are bluer than comparable-metallicity cluster main-sequence turnoffs.

Bond \& MacConnell (1971) noted that Strömgren photometry revealed two stars, BD + 251981 and BD - 122669 , that have weak metal lines and gravities consistent with dwarfs, yet temperatures hotter and colors bluer than globular cluster main-sequence turnoffs. Additional photometry and spectroscopic analysis by Carney \& Peterson (1981, hereafter CP) confirmed that the two stars are lowmetallicity field blue stragglers belonging to the halo population. CP also noted that it is important to employ kinematics in the identification of field halo blue stragglers, since there is a class of thin-disk stars, the $\lambda$ Boo stars, that manifest weak metal lines and that can mimic halo blue stragglers photometrically. These stars may be excised from a sample by requiring that the stellar kinematics be "hotter" than those of the thin disk.

Stetson (1991) has also employed $u v b y \beta$ photometry to identify stars in the solar neighborhood that are analogs to globular-cluster blue stragglers. Stetson relied on the blue 
colors, low metallicities, and high space velocities to make the identifications.

The hotter blue stragglers in several globular clusters lie within the instability strip, and stars with similar pulsational periods and low metallicities are also found in the field. These are known as SX Phe variables, and Nemec, Nemec, \& Lutz (1994) have provided a list of such stars both in the field and in globular clusters. Preston \& Landolt (1998) have recently added another to the list of field SX Phe variables: CS 22966-043. This star is particularly important, since they were able to estimate the star's metallicity $([\mathrm{Fe} / \mathrm{H}] \approx-2.4)$, its pulsation period (roughly 54 minutes), and also demonstrate that the star is a binary system, with an orbital period of 431 days and an orbital eccentricity of 0.10 . These orbital characteristics are shown in this paper to be typical of metal-poor field blue stragglers.

Carney et al. (1994, hereafter Paper XII) described a procedure to identify candidate blue stragglers: the stars should have dereddened $B-V$ color indices that are bluer than the turnoffs of comparable-metallicity globular clusters, as defined by the following equation for the main-sequence turnoff locus:

$$
(B-V)_{0, \text { turnoff }}=0.615+0.209[\mathrm{~m} / \mathrm{H}]+0.046[\mathrm{~m} / \mathrm{H}]^{2} .
$$

We restrict our study to stars with $[\mathrm{m} / \mathrm{H}] \leq-0.60$ that lie blueward of this locus. The metal-rich end of our sample extends into what is often called the "thick-disk" regime. However, we have noted earlier (Carney, Latham, \& Laird 1989) that within our sample, the thick-disk field stellar population appears to have a very sharp cutoff in $(B-V)_{0}$, consistent with the main-sequence turnoffs of the diskpopulation globular clusters 47 Tuc and M71. We concluded that the field thick-disk stars are as old as these clusters. Thus our reliance upon equation (1) should remain justified.

For this paper, we have refined the method slightly, because it is vulnerable to uncertainties in the reddening of individual field stars. We have estimated $E(B-V)$ for the program stars following the methodology outlined by Carney et al. (1987) and Laird, Carney, \& Latham (1988), which makes use of the reddening maps provided by Burstein \& Heiles (1982). We have compared our reddening estimates with those obtained from $u v b y \beta$ photometry (Schuster \& Nissen 1988; Schuster, Parrao, \& Contreras Martínez 1993), using the formulation of Schuster \& Nissen (1989). Of the 174 stars for which we could estimate $E(B-V)$ using both methods, in 134 cases $(77 \%)$ the results were the same to within $\pm 0.01 \mathrm{mag}$ (assuming that negative reddenings derived from the $u v b y \beta$ photometry indicate zero reddening). In only seven cases did the difference exceed $0.03 \mathrm{mag}$, and their average $E(B-V)$ was already large: $\geq 0.08 \mathrm{mag}$. We therefore chose as our parent sample all stars from Paper XII with $E(B-V)$ estimated by us to be $\leq 0.05 \mathrm{mag}$ and $[\mathrm{m} / \mathrm{H}] \leq-0.6$. When available, we used reddening values derived from the $u b v y \beta$ photometry. We then added six stars to the list, whose reddenings we had estimated to be larger than $0.05 \mathrm{mag}$, but for which we could derive $E(B-V)$ from the uvby $\beta$ photometry. In Figure 1 we show the resultant distribution in $(B-V)_{0}$ as a function of metallicity. The line is defined by equation (1). Since there are no clusters with $[\mathrm{Fe} / \mathrm{H}] \leq-2.2$ and avail-

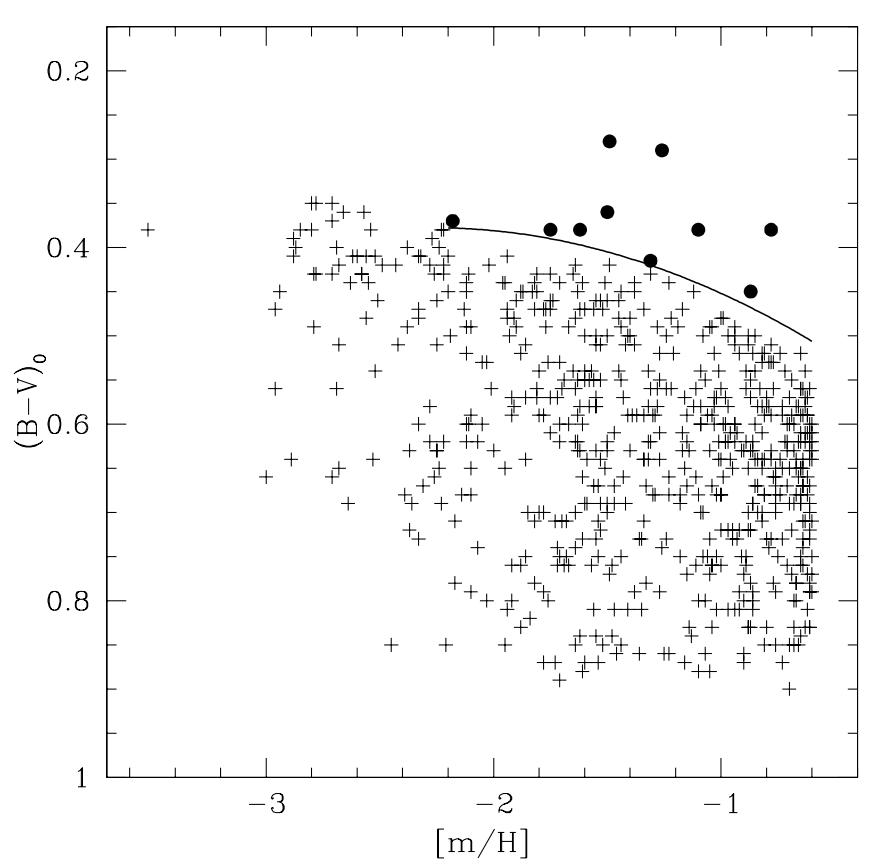

FIG. 1.-Stars of Paper XII with $-2.2 \leq[\mathrm{m} / \mathrm{H}] \leq-0.6$. The solid line represents the main-sequence turnoff locus from eq. (1). The blue-straggler candidates are plotted as filled circles.

able model isochrones do not extend to much lower metallicities, we chose to not extend the line to lower metallicities. We focus in this paper on the 10 stars with $-2.2 \leq[\mathrm{m} /$ $\mathrm{H}] \leq-0.6$ that fall above the line and are plotted as filled circles in Figure 1. We note that one of these, HD 84937, was included (barely) only because of the higher reddening estimate from the $u v b y \beta$ photometry.

In Table 1 we summarize the basic properties of these stars, including their distances and kinematics. The quantity $\Delta$ in column (1) is the difference between the observed $(B-V)_{0}$ value and that predicted by equation (1). Two values are given for the distance and stellar kinematics (Galactic U, V, and $\mathrm{W}$ velocities rounded off to the nearest $10 \mathrm{~km} \mathrm{~s}^{-1}$ ) of each star. First, we follow procedures similar to those outlined in Paper XII, except that the algorithms between absolute magnitudes, colors, and metallicities have been rederived using Hipparcos parallaxes. Because of this recalibration, the first distance for each star in Table 1 is considerably larger than the corresponding distance reported in Paper XII. For the hotter blue stragglers, this is an extrapolation beyond the calibrating stars. Even were the calibration precise, blue stragglers have a range in $M_{V}$ at a fixed color (see Bailyn 1995; his Fig. 1). The distances and kinematics are listed in the first row for each star in Table 1 using this procedure. The second row involves use of the Hipparcos parallaxes directly, although in several case the parallaxes are not well measured. The kinematics of all of our program stars are consistent with membership in the halo or old disk populations, both of which are extremely old (see Fig. 1) so that our use of equation (1) is appropriate. None of the stars have thin-disk kinematics and so are unlikely to be $\lambda$ Boo stars.

There is one additional concern in the identification of field halo or thick-disk population blue stragglers. While the bulk of the globular clusters seem to follow the locus of dereddened $B-V$ color index versus metallicity defined in 
TABLE 1

Candidate Metal-Poor Field Blue Stragglers

\begin{tabular}{|c|c|c|c|c|c|c|c|c|c|}
\hline $\begin{array}{l}\text { Star } \\
(1)\end{array}$ & $\begin{array}{l}\alpha(\mathrm{J} 2000.0) \\
\delta(\mathrm{J} 2000.0) \\
\quad(2)\end{array}$ & $\begin{array}{c}(B-V)_{0} \\
E(B-V) \\
\quad(3)\end{array}$ & $\begin{array}{c}{[\mathrm{m} / \mathrm{H}]} \\
T_{\text {eff }} \\
(4)\end{array}$ & $\begin{array}{c}M_{V} \\
\pi \text { (mas) } \\
(5)\end{array}$ & $\begin{array}{c}d \\
(\mathrm{pc}) \\
(6)\end{array}$ & $\begin{array}{l}\mathrm{U} \\
(7)\end{array}$ & $\begin{array}{l}\mathrm{V} \\
(8)\end{array}$ & $\begin{array}{l}\text { W } \\
(9)\end{array}$ & $\begin{array}{l}\text { Comments } \\
\text { (10) }\end{array}$ \\
\hline $\mathrm{BD}+7294$ & 014712.4 & 0.32 & -1.62 & 3.20 & 191 & -350 & -80 & 30 & G245-32 \\
\hline$\Delta=-0.08 \ldots \ldots$. & +732827 & 0.10 & 6620 & $6.46 \pm 1.27$ & 155 & -320 & -100 & 20 & $\mathrm{t} 6250 \mathrm{~g} 40 \mathrm{~m} 15 \mathrm{v} 004$ \\
\hline $\mathrm{BD}+401166 \ldots \ldots$ & 050528.7 & 0.42 & -0.76 & 3.66 & 158 & 140 & -140 & 160 & G96-20 \\
\hline$\Delta=-0.06 \ldots \ldots$ & +401526 & 0.00 & 6200 & $8.32 \pm 1.52$ & 120 & 130 & -100 & 130 & $\mathrm{t} 6250 \mathrm{~g} 40 \mathrm{~m} 10 \mathrm{v} 008$ \\
\hline $\mathrm{BD}+251981 \ldots \ldots$ & 084424.7 & 0.29 & -1.26 & 2.70 & 205 & 0 & -320 & -80 & \\
\hline$\Delta=-0.13 \ldots \ldots$ & +244748 & 0.01 & 6860 & $6.59 \pm 1.45$ & 152 & 10 & -240 & -50 & $\mathrm{t} 6750 \mathrm{~g} 40 \mathrm{~m} 15 \mathrm{v} 010$ \\
\hline BD $-122669 \ldots \ldots$ & 084639.6 & 0.28 & -1.49 & 2.73 & 309 & 180 & -100 & -460 & $\ldots$ \\
\hline$\Delta=-0.13 \ldots \ldots$. & -132125 & 0.02 & 6940 & $5.76 \pm 1.50$ & 174 & 110 & -60 & -240 & $\mathrm{t} 7000 \mathrm{~g} 40 \mathrm{~m} 15 \mathrm{v} 030$ \\
\hline HD $84937 \ldots \ldots \ldots$ & 094856.1 & 0.37 & -2.18 & 4.05 & 70 & -210 & -200 & 0 & G43-3 \\
\hline$\Delta=-0.01 \ldots \ldots$ & +134439 & 0.02 & 6300 & $12.44 \pm 1.06$ & 80 & -240 & -230 & 0 & $\mathrm{t} 6250 \mathrm{~g} 40 \mathrm{~m} 20 \mathrm{v} 004$ \\
\hline HD $97916 \ldots \ldots \ldots$ & 111554.2 & 0.415 & -1.31 & 3.64 & 129 & -120 & 20 & 110 & $\ldots$ \\
\hline$\Delta=-0.005 \ldots \ldots$ & +020512 & 0.01 & 6250 & $7.69 \pm 1.23$ & 130 & -120 & 20 & 110 & $\mathrm{t} 6250 \mathrm{~g} 40 \mathrm{~m} 15 \mathrm{v} 012$ \\
\hline HD $106516 \ldots \ldots \ldots$ & 121510.6 & 0.45 & -0.87 & 3.88 & 28 & -80 & -70 & -70 & $\ldots$ \\
\hline$\Delta=-0.02 \ldots \ldots$ & -101845 & 0.00 & 6120 & $44.34 \pm 1.01$ & 23 & -70 & -60 & -60 & $\mathrm{t} 6000 \mathrm{~g} 40 \mathrm{~m} 10 \mathrm{v} 010$ \\
\hline $\mathrm{BD}+511817 \ldots \ldots$ & 130839.1 & 0.38 & -1.10 & $3 . \overline{44}$ & 228 & -90 & -250 & 60 & G177-23 \\
\hline$\Delta=-0.06 \ldots \ldots$ & +510359 & 0.00 & 6330 & $6.22 \pm 1.46$ & 161 & -70 & -180 & 30 & $\mathrm{t} 6250 \mathrm{~g} 40 \mathrm{~m} 10 \mathrm{v} 008$ \\
\hline G66-30 ............ & 145007.8 & 0.37 & -1.75 & 3.82 & 267 & 200 & -300 & 10 & $\ldots$ \\
\hline$\Delta=-0.02 \ldots \ldots$ & +005027 & 0.03 & 6280 & $7.01 \pm 2.43$ & 143 & 140 & -150 & -30 & $\mathrm{t} 6250 \mathrm{~g} 40 \mathrm{~m} 15 \mathrm{v} 006$ \\
\hline G202-65 ........... & 163558.6 & 0.36 & -1.50 & 3.10 & 421 & 430 & -230 & 50 & $\ldots$ \\
\hline$\Delta=-0.05 \ldots \ldots$ & +455159 & 0.00 & 6560 & $2.22 \pm 1.48$ & 450 & 450 & -230 & 60 & $\mathrm{t} 6500 \mathrm{~g} 40 \mathrm{~m} 15 \mathrm{v} 010$ \\
\hline
\end{tabular}

NoтE.- Units of right ascension are hours, minutes, and seconds, and units of declination are degrees, arcminutes, and arcseconds.

Paper XII, there are some exceptions. Ruprecht 106, for example, is a "young" globular cluster, with $[\mathrm{Fe} /$ $\mathrm{H}] \approx-1.5$ (Brown, Wallerstein, \& Zucker 1996) and a dereddened $B-V$ turnoff color index of $0.33 \mathrm{mag}$ (Buonanno et al. 1993), whereas the algorithm of Paper XII would have predicted 0.40 mag. Further, Preston, Beers, \& Shectman (1994) have identified a field population of metalpoor stars that appear to be main-sequence stars, but extending to much bluer colors than the turnoff locus of Figure 1. Preston et al. (1994) suggested that these stars may be the result of a merger into the Milky Way of a small galaxy having a larger fraction of young but metal-poor stars. If this is the correct explanation, then some of the victim's main-sequence stars could masquerade as halo blue stragglers, ironically mimicking one of the early explanations of the blue stragglers - delayed star formation. Carney et al. (1996) estimated the number of such stars within the sample in Paper XII from which our program stars have been obtained. They estimated that perhaps one of their blue-straggler candidates could have originated in the victim galaxy. Even this may be an overestimate, since Preston \& Sneden (2000) have argued that some of their "blue metal-poor" stars are Galactic blue stragglers rather than young metal-poor stars captured from a satellite galaxy.

\section{OBSERVATIONS}

The radial velocities of our 10 candidate field blue stragglers were monitored with the Center for Astrophysics (CfA) digital speedometers (Latham 1985, 1992). Three nearly identical instruments were used on the Multiple Mirror Telescope and $1.5 \mathrm{~m}$ Tillinghast Reflector at the Whipple Observatory atop Mount Hopkins, Arizona, and on the 1.5 m Wyeth Reflector located in Harvard, Massachusetts. Echelle spectrographs were used with intensified photoncounting Reticon detectors to record about $45 \AA$ of spec- trum in a single order centered near $5187 \AA$, with a resolution of about $8.3 \mathrm{~km} \mathrm{~s}^{-1}$ and signal-to-noise ratios (S/Ns) ranging from 7 to 50 per resolution element.

Radial velocities were measured from the observed spectra using the one-dimensional correlation package RVSAO (Kurtz \& Mink 1998) running inside the IRAF ${ }^{3}$ environment. For the templates we used a new grid of synthetic spectra (Morse \& Kurucz 2001) calculated using the model atmospheres computed using Kurucz's code ATLAS9. The new grid of synthetic templates incorporates several improvements compared with the older grid that we have been using for several years (for example, see Nordström et al. 1994). Considerable effort was invested in the development of a revised line list for the wavelength region 5146 to $5229 \AA$, with careful verification that the calculated spectra matched the high-quality observed flux spectrum available for the Sun (Kurucz et al. 1984). For all models used here, scaled solar abundances were employed. The new grid extends the wavelength coverage so that it now covers the entire range of observed wavelengths for any radial velocity likely to be encountered. (The current range in $V_{r}$ seen in Galactic field stars extends from -583 $\mathrm{km} \mathrm{s}^{-1}$ for G233-27 to $+546 \mathrm{~km} \mathrm{~s}^{-1}$ for CD - 292277 .) In addition, the new grid extends the range of metallicities (typically $[\mathrm{m} / \mathrm{H}]=-4.0$ to +0.5 , in intervals of 0.5 dex), gravities ( $\log g=0.5$ to 5.0 in intervals of $0.5 \mathrm{dex}$ ), and effective temperatures $\left(T_{\text {eff }}=3500\right.$ to $12,000 \mathrm{~K}$, in intervals of $250 \mathrm{~K}$ up to $10,000 \mathrm{~K}$ and $500 \mathrm{~K}$ above that). With the further goal of deriving more reliable rotational velocities, especially for the values below $10 \mathrm{~km} \mathrm{~s}^{-1}$, the new grid of templates is more finely divided in rotational velocity, with

\footnotetext{
${ }^{3}$ IRAF is distributed by the National Optical Astronomy Observatory, which is operated by the Association of Universities for Research in Astronomy, Inc., under cooperative agreement with the National Science Foundation.
} 
TABLE 2

Stellar Radial Velocities

\begin{tabular}{|c|c|c|c|c|c|c|c|c|}
\hline HJD & $V_{r}$ & $\sigma$ & HJD & $V_{r}$ & $\sigma$ & HJD & $V_{r}$ & $\sigma$ \\
\hline & & & $\mathrm{BD}+7294$ & 014712.4 & +732827 & & & \\
\hline $45605.932 \ldots \ldots$ & -268.85 & 0.87 & $48877.840 \ldots \ldots$ & -270.47 & 0.89 & $50652.955 \ldots \ldots$ & -269.30 & 0.72 \\
\hline $45720.715 \ldots \ldots$ & -269.08 & 0.61 & $48907.873 \ldots \ldots$ & -267.64 & 0.86 & $51507.737 \ldots \ldots$ & -268.38 & 0.86 \\
\hline $46018.820 \ldots \ldots$ & -266.63 & 0.91 & $49264.849 \ldots \ldots$ & -268.10 & 0.80 & $51533.743 \ldots \ldots$ & -268.26 & 0.81 \\
\hline $46337.893 \ldots \ldots$ & -269.02 & 0.82 & $49295.806 \ldots \ldots$ & -267.38 & 0.83 & $51595.590 \ldots \ldots$ & -269.35 & 0.84 \\
\hline $46397.703 \ldots \ldots$ & -269.76 & 1.09 & $49317.709 \ldots \ldots$ & -267.54 & 1.01 & $51833.894 \ldots \ldots$ & -268.04 & 0.74 \\
\hline $46452.638 \ldots \ldots$ & -268.75 & 0.88 & $49347.667 \ldots \ldots$ & -267.68 & 0.74 & $51857.758 \ldots \ldots$ & -270.18 & 0.92 \\
\hline $46723.864 \ldots \ldots$ & -269.00 & 0.67 & $49558.986 \ldots \ldots$ & -268.29 & 1.01 & $51861.756 \ldots \ldots$ & -269.74 & 0.68 \\
\hline $47053.947 \ldots \ldots$ & -267.15 & 0.66 & $49613.829 \ldots \ldots$ & -269.14 & 0.99 & $51886.786 \ldots \ldots$ & -268.87 & 0.66 \\
\hline $47369.994 \ldots \ldots$ & -268.31 & 0.94 & $49644.740 \ldots \ldots$ & -269.53 & 0.87 & $51947.619 \ldots \ldots$ & -268.44 & 0.73 \\
\hline $47728.989 \ldots \ldots$ & -268.29 & 0.81 & $49650.752 \ldots \ldots$ & -267.88 & 0.88 & & & \\
\hline \multirow{2}{*}{$47958.593 \ldots \ldots$} & -267.77 & 0.60 & $49678.696 \ldots \ldots$ & -268.88 & 0.96 & & & \\
\hline & & & $\mathrm{BD}+401166$ & 050528.7 & +401526 & & & \\
\hline $45063.629 \ldots \ldots$ & 105.64 & 0.64 & $47019.844 \ldots$ & 105.80 & 0.77 & $51535.780 \ldots$ & 104.23 & 0.65 \\
\hline $45250.858 \ldots \ldots$ & 106.67 & 0.84 & $47433.842 \ldots \ldots$ & 105.21 & 0.70 & $51571.619 \ldots \ldots$ & 105.51 & 0.78 \\
\hline $45689.711 \ldots \ldots$ & 105.69 & 0.76 & $47813.758 \ldots \ldots$ & 105.43 & 0.76 & $51610.545 \ldots \ldots$ & 105.83 & 0.69 \\
\hline $46007.879 \ldots \ldots$ & 104.47 & 0.93 & $48170.811 \ldots \ldots$ & 106.24 & 0.72 & $51806.795 \ldots \ldots$ & 105.69 & 0.66 \\
\hline $46127.599 \ldots \ldots$ & 105.08 & 0.79 & $48642.651 \ldots \ldots$ & 106.03 & 0.62 & $51831.942 \ldots \ldots$ & 104.96 & 0.49 \\
\hline $46345.876 \ldots \ldots$ & 105.60 & 0.64 & $48991.664 \ldots \ldots$ & 105.79 & 0.74 & $51871.842 \ldots \ldots$ & 106.73 & 0.78 \\
\hline $46478.589 \ldots \ldots$ & 104.51 & 0.78 & $50884.622 \ldots \ldots$ & 105.25 & 0.72 & $51902.734 \ldots \ldots$ & 105.61 & 0.67 \\
\hline $46698.878 \ldots \ldots$ & 105.26 & 0.66 & $51268.674 \ldots \ldots$ & 106.09 & 0.55 & $51948.674 \ldots \ldots$ & 106.16 & 0.71 \\
\hline \multirow[t]{2}{*}{$46715.808 \ldots \ldots$} & 105.49 & 0.72 & $51467.804 \ldots \ldots$ & 104.01 & 0.63 & $51994.586 \ldots \ldots$ & 104.44 & 0.80 \\
\hline & & & $\mathrm{BD}+251981$ & 084424.7 & +244748 & & & \\
\hline $46193.551 \ldots \ldots$ & 57.11 & 0.53 & $47581.701 \ldots \ldots$ & 56.30 & 0.50 & $50770.991 \ldots \ldots$ & 58.15 & 0.38 \\
\hline $46196.531 \ldots \ldots$ & 57.05 & 0.58 & $47599.615 \ldots \ldots$ & 57.35 & 0.74 & $50793.941 \ldots \ldots$ & 58.03 & 0.56 \\
\hline $46359.886 \ldots \ldots$ & 57.45 & 0.43 & $47611.666 \ldots \ldots$ & 57.98 & 0.52 & $50822.876 \ldots \ldots$ & 57.19 & 0.48 \\
\hline $46402.877 \ldots \ldots$ & 58.35 & 0.65 & $47634.726 \ldots \ldots$ & 56.96 & 0.49 & $50885.728 \ldots \ldots$ & 57.18 & 0.43 \\
\hline $46409.826 \ldots \ldots$ & 57.53 & 0.53 & $47899.762 \ldots \ldots$ & 56.10 & 0.61 & $50915.801 \ldots \ldots$ & 57.59 & 0.58 \\
\hline $46427.826 \ldots \ldots$ & 57.63 & 0.51 & $47935.571 \ldots \ldots$ & 56.54 & 0.58 & $50948.644 \ldots \ldots$ & 59.17 & 0.60 \\
\hline $46452.721 \ldots \ldots$ & 57.79 & 0.48 & $47956.606 \ldots \ldots$ & 57.45 & 0.56 & $51151.018 \ldots \ldots$ & 58.01 & 0.41 \\
\hline $46478.679 \ldots \ldots$ & 57.17 & 0.66 & $47989.587 \ldots \ldots$ & 57.16 & 0.56 & $51178.960 \ldots \ldots$ & 58.04 & 0.49 \\
\hline $46491.597 \ldots \ldots$ & 57.17 & 0.57 & $48053.627 \ldots \ldots$ & 57.41 & 0.64 & $51233.746 \ldots \ldots$ & 57.70 & 0.49 \\
\hline $46512.562 \ldots \ldots$ & 56.75 & 0.65 & $48201.042 \ldots \ldots$ & 57.81 & 0.55 & $51268.793 \ldots \ldots$ & 57.93 & 0.43 \\
\hline $46536.545 \ldots \ldots$ & 55.67 & 1.11 & $48286.798 \ldots \ldots$ & 57.27 & 0.39 & $51295.765 \ldots \ldots$ & 58.48 & 0.41 \\
\hline $46600.635 \ldots \ldots$ & 57.75 & 0.49 & $48409.634 \ldots \ldots$ & 58.73 & 0.59 & $51503.052 \ldots \ldots$ & 57.58 & 0.36 \\
\hline $46833.721 \ldots \ldots$ & 57.36 & 0.59 & $48872.001 \ldots \ldots$ & 57.00 & 0.48 & $51533.955 \ldots \ldots$ & 57.45 & 0.42 \\
\hline $46862.685 \ldots \ldots$ & 57.65 & 0.60 & $48912.033 \ldots \ldots$ & 58.82 & 0.50 & $51567.898 \ldots \ldots$ & 56.50 & 0.53 \\
\hline $46897.589 \ldots \ldots$ & 58.17 & 0.52 & $48931.990 \ldots \ldots$ & 56.91 & 0.56 & $51622.800 \ldots \ldots$ & 57.38 & 0.29 \\
\hline $47106.923 \ldots \ldots$ & 56.70 & 0.41 & $48965.961 \ldots \ldots$ & 58.63 & 0.46 & $51653.776 \ldots \ldots$ & 57.93 & 0.48 \\
\hline $47132.879 \ldots \ldots$ & 57.92 & 0.55 & $48991.863 \ldots \ldots$ & 57.83 & 0.47 & $51856.007 \ldots \ldots$ & 57.02 & 0.34 \\
\hline $47133.914 \ldots \ldots$ & 56.14 & 0.51 & $49021.890 \ldots \ldots$ & 57.01 & 0.40 & $51884.022 \ldots \ldots$ & 58.25 & 0.46 \\
\hline $47462.938 \ldots \ldots$ & 55.81 & 0.77 & $49056.813 \ldots \ldots$ & 58.38 & 0.56 & $51920.959 \ldots \ldots$ & 58.36 & 0.42 \\
\hline $47488.915 \ldots \ldots$ & 56.12 & 0.50 & $49078.711 \ldots \ldots$ & 57.86 & 0.58 & $51944.897 \ldots \ldots$ & 57.88 & 0.43 \\
\hline $47515.838 \ldots \ldots$ & 56.64 & 0.69 & $49111.613 \ldots \ldots$ & 58.80 & 0.38 & $51971.791 \ldots \ldots$ & 57.99 & 0.54 \\
\hline \multirow[t]{2}{*}{$47540.829 \ldots \ldots$} & 55.88 & 0.57 & $49291.979 \ldots \ldots$ & 57.65 & 0.42 & $52006.746 \ldots \ldots$ & 57.69 & 0.46 \\
\hline & & & BD -12 2669 & 084639.6 & -132125 & & & \\
\hline $46193.539 \ldots \ldots$ & 44.85 & 3.04 & $46847.694 \ldots \ldots$ & 45.67 & 1.78 & $47280.631 \ldots \ldots$ & 49.31 & 1.27 \\
\hline $46196.546 \ldots \ldots$ & 52.85 & 2.90 & $46861.737 \ldots \ldots$ & 42.39 & 1.41 & $47284.651 \ldots \ldots$ & 51.44 & 1.35 \\
\hline $46404.834 \ldots \ldots$ & 35.02 & 2.12 & $46862.732 \ldots \ldots$ & 46.75 & 1.25 & $47287.685 \ldots \ldots$ & 52.16 & 1.43 \\
\hline $46409.860 \ldots \ldots$ & 37.12 & 2.37 & $46863.784 \ldots \ldots$ & 45.72 & 1.43 & $47307.639 \ldots \ldots$ & 50.45 & 1.47 \\
\hline $46421.852 \ldots \ldots$ & 37.10 & 2.08 & $46864.835 \ldots \ldots$ & 44.61 & 3.57 & $47423.008 \ldots \ldots$ & 39.73 & 1.19 \\
\hline $46427.791 \ldots \ldots$ & 33.60 & 1.81 & $46865.719 \ldots \ldots$ & 49.04 & 1.74 & $47430.020 \ldots \ldots$ & 39.96 & 1.73 \\
\hline $46428.747 \ldots \ldots$ & 39.07 & 1.73 & $46866.781 \ldots \ldots$ & 46.77 & 1.55 & $47459.027 \ldots \ldots$ & 35.79 & 1.45 \\
\hline $46452.861 \ldots \ldots$ & 44.00 & 0.79 & $46867.807 \ldots \ldots$ & 44.43 & 1.39 & $47467.006 \ldots \ldots$ & 35.62 & 1.43 \\
\hline $46453.849 \ldots \ldots$ & 44.62 & 0.97 & $46889.712 \ldots \ldots$ & 47.79 & 1.66 & $47484.958 \ldots \ldots$ & 32.20 & 1.66 \\
\hline $46454.854 \ldots \ldots$ & 42.02 & 1.26 & $46891.711 \ldots \ldots$ & 50.62 & 1.70 & $47486.030 \ldots \ldots$ & 32.88 & 1.53 \\
\hline $46455.867 \ldots \ldots$ & 40.50 & 1.79 & $46892.722 \ldots \ldots$ & 52.34 & 2.15 & $47486.942 \ldots \ldots$ & 33.00 & 1.89 \\
\hline $46456.863 \ldots \ldots$ & 39.17 & 1.67 & $46894.682 \ldots \ldots$ & 50.77 & 1.42 & $47493.023 \ldots \ldots$ & 32.99 & 1.31 \\
\hline $46457.821 \ldots \ldots$ & 43.37 & 1.50 & $46895.651 \ldots \ldots$ & 48.82 & 1.86 & $47543.829 \ldots \ldots$ & 34.44 & 1.73 \\
\hline $46458.827 \ldots \ldots$ & 44.92 & 1.64 & $46921.647 \ldots \ldots$ & 51.91 & 1.89 & $47550.886 \ldots \ldots$ & 34.67 & 1.24 \\
\hline $46486.749 \ldots \ldots$ & 48.58 & 1.30 & $46925.658 \ldots \ldots$ & 51.00 & 1.11 & $47554.888 \ldots \ldots$ & 32.20 & 1.54 \\
\hline
\end{tabular}


TABLE 2-Continued

\begin{tabular}{|c|c|c|c|c|c|c|c|c|}
\hline HJD & $V_{r}$ & $\sigma$ & HJD & $V_{r}$ & $\sigma$ & HJD & $V_{r}$ & $\sigma$ \\
\hline $46487.721 \ldots \ldots$ & 41.57 & 1.64 & $47077.024 \ldots \ldots$ & 36.72 & 1.58 & $47573.814 \ldots \ldots$ & 35.67 & 1.54 \\
\hline $46488.804 \ldots \ldots$ & 46.07 & 2.08 & $47110.016 \ldots \ldots$ & 33.01 & 2.08 & $47577.825 \ldots \ldots$ & 32.13 & 2.59 \\
\hline $46489.798 \ldots \ldots$ & 45.33 & 1.49 & $47111.018 \ldots \ldots$ & 34.39 & 1.51 & $47580.724 \ldots \ldots$ & 36.00 & 1.39 \\
\hline $46509.690 \ldots \ldots$ & 47.49 & 2.09 & $47111.972 \ldots \ldots$ & 33.09 & 1.93 & $47583.698 \ldots \ldots$ & 33.71 & 1.34 \\
\hline $46510.784 \ldots \ldots$ & 49.70 & 2.26 & $47131.936 \ldots \ldots$ & 33.99 & 1.01 & $47600.688 \ldots \ldots$ & 41.53 & 1.35 \\
\hline $46511.752 \ldots \ldots$ & 51.81 & 1.94 & $47133.010 \ldots \ldots$ & 35.57 & 1.99 & $47601.798 \ldots \ldots$ & 41.57 & 1.81 \\
\hline $46514.692 \ldots \ldots$ & 51.37 & 1.24 & $47133.893 \ldots \ldots$ & 33.30 & 0.98 & $47604.672 \ldots \ldots$ & 41.11 & 1.31 \\
\hline $46541.664 \ldots \ldots$ & 50.32 & 1.31 & $47134.952 \ldots \ldots$ & 33.32 & 1.38 & $47610.635 \ldots \ldots$ & 44.18 & 1.29 \\
\hline $46567.626 \ldots \ldots$ & 52.30 & 1.57 & $47135.948 \ldots \ldots$ & 32.96 & 1.34 & $47614.726 \ldots \ldots$ & 40.33 & 1.55 \\
\hline $46568.625 \ldots \ldots$ & 49.92 & 1.19 & $47136.991 \ldots \ldots$ & 33.62 & 1.49 & $47632.651 \ldots \ldots$ & 45.63 & 1.59 \\
\hline $46569.634 \ldots \ldots$ & 48.92 & 1.27 & $47137.921 \ldots \ldots$ & 32.51 & 1.18 & $47637.669 \ldots \ldots$ & 43.97 & 1.90 \\
\hline $46723.033 \ldots \ldots$ & 29.51 & 1.58 & $47138.955 \ldots \ldots$ & 31.88 & 1.32 & $47877.981 \ldots \ldots$ & 35.48 & 1.71 \\
\hline $46724.029 \ldots \ldots$ & 30.69 & 1.35 & $47139.961 \ldots \ldots$ & 33.90 & 1.71 & $47878.850 \ldots \ldots$ & 33.60 & 1.70 \\
\hline $46725.027 \ldots \ldots$ & 31.56 & 1.16 & $47140.974 \ldots \ldots$ & 33.87 & 1.16 & $47901.807 \ldots \ldots$ & 34.10 & 1.31 \\
\hline $46755.008 \ldots \ldots$ & 33.32 & 1.05 & $47158.949 \ldots \ldots$ & 35.34 & 1.16 & $47905.921 \ldots \ldots$ & 31.47 & 1.28 \\
\hline $46774.013 \ldots \ldots$ & 35.43 & 1.67 & $47159.853 \ldots \ldots$ & 33.61 & 1.23 & $47929.762 \ldots \ldots$ & 36.77 & 1.75 \\
\hline $46779.945 \ldots \ldots$ & 30.57 & 1.67 & $47163.902 \ldots \ldots$ & 33.11 & 1.25 & $47957.757 \ldots \ldots$ & 35.35 & 1.44 \\
\hline $46783.918 \ldots \ldots$ & 33.75 & 1.80 & $47191.789 \ldots \ldots$ & 34.01 & 1.21 & $47984.730 \ldots \ldots$ & 38.55 & 1.60 \\
\hline $46814.865 \ldots \ldots$ & 37.12 & 1.79 & $47192.814 \ldots \ldots$ & 36.67 & 1.57 & $47985.696 \ldots \ldots$ & 40.98 & 1.44 \\
\hline $46816.939 \ldots \ldots$ & 37.33 & 1.60 & $47219.772 \ldots \ldots$ & 42.85 & 1.33 & $47987.685 \ldots \ldots$ & 41.36 & 1.28 \\
\hline $46817.787 \ldots \ldots$ & 36.95 & 1.68 & 47224.702 . & 40.97 & 1.47 & $47992.684 \ldots \ldots$ & 41.56 & 1.61 \\
\hline $46834.803 \ldots \ldots$ & 43.16 & 2.37 & 47225.702. & 43.71 & 1.50 & $47997.668 \ldots \ldots$ & 43.45 & 1.77 \\
\hline $46835.900 \ldots \ldots$ & 41.36 & 1.38 & $47229.669 \ldots \ldots$ & 44.41 & 1.33 & $48018.652 \ldots \ldots$ & 47.75 & 1.71 \\
\hline $46836.743 \ldots \ldots$ & 38.96 & 1.47 & $47230.657 \ldots \ldots$ & 43.34 & 1.33 & $48205.029 \ldots \ldots$ & 39.81 & 1.75 \\
\hline $46837.764 \ldots \ldots$ & 40.83 & 1.34 & $47249.705 \ldots \ldots$ & 43.84 & 2.65 & $48256.881 \ldots \ldots$ & 29.92 & 1.37 \\
\hline $46838.715 \ldots \ldots$ & 41.39 & 1.03 & $47249.738 \ldots \ldots$ & 46.91 & 1.48 & $48281.826 \ldots \ldots$ & 34.22 & 1.26 \\
\hline $46839.717 \ldots \ldots$ & 44.10 & 1.74 & $47250.662 \ldots \ldots$ & 46.13 & 1.40 & $48282.783 \ldots \ldots$ & 33.49 & 1.63 \\
\hline $46842.712 \ldots \ldots$ & 42.62 & 1.79 & $47252.672 \ldots \ldots$ & 46.56 & 1.38 & $48284.888 \ldots \ldots$ & 33.34 & 1.78 \\
\hline $46844.770 \ldots \ldots$ & 40.79 & 2.05 & $47254.719 \ldots \ldots$ & 45.64 & 1.21 & $48289.824 \ldots \ldots$ & 31.76 & 1.01 \\
\hline \multirow[t]{2}{*}{$46845.743 \ldots \ldots$} & 45.11 & 1.66 & $47278.640 \ldots \ldots$ & 50.73 & 1.04 & $48290.893 \ldots \ldots$ & 31.32 & 1.13 \\
\hline & & & HD 84937 & 094856.1 & +134439 & & & \\
\hline $45035.785 \ldots \ldots$ & -13.95 & 0.68 & $47215.848 \ldots \ldots$ & -14.93 & 0.57 & $49493.603 \ldots \ldots$ & -16.13 & 0.50 \\
\hline $45696.899 \ldots \ldots$ & -14.21 & 0.82 & $47527.853 \ldots \ldots$ & -15.01 & 0.70 & $49506.573 \ldots \ldots$ & -14.89 & 0.66 \\
\hline $46012.945 \ldots \ldots$ & -12.87 & 0.80 & $47542.739 \ldots \ldots$ & -16.10 & 0.60 & $49637.892 \ldots \ldots$ & -16.74 & 0.76 \\
\hline $46128.535 \ldots \ldots$ & -14.84 & 0.87 & $47578.642 \ldots \ldots$ & -12.52 & 1.10 & $49643.919 .$. & -15.92 & 0.78 \\
\hline $46155.605 \ldots \ldots$ & -15.18 & 0.84 & $47605.594 \ldots \ldots$ & -14.75 & 0.70 & $49652.926 \ldots \ldots$ & -16.45 & 0.66 \\
\hline $46221.570 \ldots \ldots$ & -15.40 & 0.98 & $47639.675 \ldots \ldots$ & -14.16 & 0.54 & $49697.008 \ldots \ldots$ & -15.48 & 0.44 \\
\hline $46361.932 \ldots \ldots$ & -14.85 & 0.81 & $47698.637 \ldots \ldots$ & -14.51 & 0.49 & $49700.052 \ldots \ldots$ & -15.12 & 0.56 \\
\hline $46402.962 \ldots \ldots$ & -14.57 & 0.64 & $48260.742 \ldots \ldots$ & -13.41 & 1.67 & $49702.972 \ldots \ldots$ & -15.91 & 0.54 \\
\hline $46425.895 \ldots \ldots$ & -13.99 & 0.60 & $48607.828 \ldots \ldots$ & -14.62 & 0.70 & $49704.947 \ldots \ldots$ & -16.51 & 0.54 \\
\hline $46448.826 \ldots \ldots$ & -16.61 & 0.79 & $48940.924 \ldots \ldots$ & -15.97 & 0.41 & $50788.943 \ldots \ldots$ & -15.41 & 0.64 \\
\hline $46453.800 \ldots \ldots$ & -16.12 & 0.74 & $49285.889 \ldots \ldots$ & -14.84 & 0.71 & $50884.750 \ldots \ldots$ & -15.14 & 0.78 \\
\hline $46455.746 \ldots \ldots$ & -13.40 & 0.86 & $49316.907 \ldots \ldots$ & -16.89 & 0.92 & $50914.687 \ldots \ldots$ & -15.51 & 0.67 \\
\hline $46478.625 \ldots \ldots$ & -15.49 & 0.82 & $49321.873 \ldots \ldots$ & -15.89 & 0.77 & $50949.535 \ldots \ldots$ & -14.94 & 0.69 \\
\hline $46488.656 \ldots \ldots$ & -16.06 & 0.57 & $49344.859 \ldots \ldots$ & -15.13 & 0.62 & $51268.782 \ldots \ldots$ & -14.63 & 0.36 \\
\hline $46490.630 \ldots \ldots$ & -15.08 & 0.75 & $49361.859 \ldots \ldots$ & -15.97 & 0.59 & $51514.930 \ldots \ldots$ & -15.66 & 1.05 \\
\hline $46492.656 \ldots \ldots$ & -15.44 & 0.62 & $49371.800 \ldots \ldots$ & -15.68 & 0.58 & $51544.933 \ldots \ldots$ & -16.20 & 0.95 \\
\hline $46508.589 \ldots \ldots$ & -15.94 & 0.64 & $49377.803 \ldots \ldots$ & -16.11 & 0.66 & $51576.769 \ldots \ldots$ & -16.31 & 0.70 \\
\hline $46513.494 \ldots \ldots$ & -15.27 & 0.60 & $49386.797 \ldots \ldots$ & -15.98 & 0.58 & $51610.745 \ldots \ldots$ & -15.11 & 0.55 \\
\hline $46536.570 \ldots \ldots$ & -15.40 & 0.59 & $49400.747 \ldots \ldots$ & -16.03 & 0.56 & $51640.584 \ldots \ldots$ & -15.41 & 0.61 \\
\hline $46581.559 \ldots \ldots$ & -15.31 & 0.60 & $49410.808 \ldots \ldots$ & -16.81 & 0.64 & $51690.569 \ldots \ldots$ & -14.75 & 0.56 \\
\hline $46600.629 \ldots \ldots$ & -15.55 & 0.27 & $49417.758 \ldots \ldots$ & -16.15 & 0.79 & $51869.948 \ldots \ldots$ & -15.57 & 0.72 \\
\hline $46757.956 \ldots \ldots$ & -14.36 & 0.92 & $49429.667 \ldots \ldots$ & -16.94 & 0.62 & $51899.824 \ldots \ldots$ & -13.85 & 0.75 \\
\hline $46770.895 \ldots \ldots$ & -15.04 & 0.77 & $49437.690 \ldots \ldots$ & -16.05 & 0.46 & $51933.799 \ldots \ldots$ & -15.28 & 0.74 \\
\hline $46812.791 \ldots \ldots$ & -15.22 & 0.76 & $49462.635 \ldots \ldots$ & -16.14 & 0.79 & $51979.740 \ldots \ldots$ & -15.88 & 0.57 \\
\hline $47166.827 \ldots \ldots$ & -14.09 & 0.95 & $49466.640 \ldots \ldots$ & -15.57 & 0.76 & & & \\
\hline \multirow[t]{2}{*}{$47189.733 \ldots \ldots$} & -16.13 & 0.97 & $49476.631 \ldots \ldots$ & -15.47 & 0.82 & & & \\
\hline & & & HD 97916 & 111554.2 & +020512 & & & \\
\hline $45784.710 \ldots \ldots$ & 64.00 & 0.62 & $46425.947 .$. & 66.38 & 0.53 & $46890.787 \ldots \ldots$ & 66.06 & 0.49 \\
\hline $46071.825 \ldots \ldots$ & 53.96 & 0.82 & $46491.722 \ldots \ldots$ & 61.60 & 0.64 & $46896.628 \ldots \ldots$ & 65.54 & 0.69 \\
\hline $46130.592 \ldots \ldots$ & 59.90 & 1.21 & $46512.620 \ldots \ldots$ & 59.55 & 0.59 & $46918.558 \ldots \ldots$ & 65.65 & 0.82 \\
\hline $46154.728 \ldots \ldots$ & 61.23 & 0.86 & $46540.597 \ldots \ldots$ & 57.60 & 0.52 & $46931.616 \ldots \ldots$ & 66.95 & 0.63 \\
\hline $46186.561 \ldots \ldots$ & 63.09 & 0.69 & $46581.600 \ldots \ldots$ & 54.85 & 0.67 & $46955.683 \ldots \ldots$ & 68.71 & 0.47 \\
\hline
\end{tabular}


TABLE 2-Continued

\begin{tabular}{|c|c|c|c|c|c|c|c|c|}
\hline HJD & $V_{r}$ & $\sigma$ & HJD & $V_{r}$ & $\sigma$ & HJD & $V_{r}$ & $\sigma$ \\
\hline $46193.599 \ldots \ldots$ & 63.65 & 0.58 & $46601.635 \ldots \ldots$ & 54.44 & 0.47 & $46988.627 \ldots$ & 69.30 & 0.48 \\
\hline $46196.565 \ldots \ldots$ & 62.95 & 0.72 & $46771.959 \ldots \ldots$ & 57.73 & 0.69 & $48960.956 \ldots \ldots$ & 68.68 & 0.66 \\
\hline $46197.606 \ldots \ldots$ & 63.88 & 0.77 & $46809.828 \ldots \ldots$ & 60.95 & 0.51 & $50826.042 \ldots \ldots$ & 62.35 & 0.47 \\
\hline $46220.582 \ldots \ldots$ & 65.52 & 0.58 & $46816.908 \ldots \ldots$ & 60.83 & 0.48 & 50846.728 . & 63.78 & 0.48 \\
\hline $46404.888 \ldots \ldots$ & 67.31 & 0.54 & $46831.820 \ldots \ldots$ & 61.64 & 0.55 & $50867.788 \ldots$ & 66.57 & 0.55 \\
\hline \multirow[t]{2}{*}{$46418.962 \ldots \ldots$} & 66.12 & 0.85 & $46862.788 \ldots \ldots$ & 63.81 & 0.57 & & & \\
\hline & & & HD 106516 & 121510.6 & -101845 & & & \\
\hline $45695.939 \ldots \ldots$ & 1.81 & 0.48 & $46554.615 \ldots \ldots$ & 1.69 & 0.44 & $47136.970 \ldots \ldots$ & 11.52 & 0.41 \\
\hline $45717.801 \ldots \ldots$ & 0.64 & 0.45 & $46568.594 \ldots \ldots$ & -0.03 & 0.29 & $47163.986 \ldots \ldots$ & 11.66 & 0.42 \\
\hline $46131.822 \ldots \ldots$ & 8.45 & 0.40 & $46598.578 \ldots \ldots$ & -1.42 & 0.62 & $47187.819 \ldots \ldots$ & 11.81 & 0.62 \\
\hline $46154.736 \ldots \ldots$ & 8.82 & 0.51 & $46770.925 \ldots$ & -2.16 & 0.52 & $47202.880 \ldots \ldots$ & 10.28 & 0.56 \\
\hline $46193.654 \ldots \ldots$ & 11.15 & 0.32 & $46785.973 \ldots$ & -2.28 & 0.40 & $47215.909 \ldots \ldots$ & 9.95 & 0.75 \\
\hline $46406.961 \ldots \ldots$ & 9.40 & 0.25 & $46800.925 \ldots$ & -1.52 & 0.39 & $47218.935 \ldots \ldots$ & 11.00 & 0.76 \\
\hline $46421.935 \ldots \ldots$ & 7.95 & 0.38 & $46834.804 \ldots \ldots$ & -0.28 & 0.41 & $47227.734 \ldots \ldots$ & 9.28 & 0.36 \\
\hline $46425.981 \ldots \ldots$ & 8.25 & 0.41 & $46863.769 \ldots \ldots$ & 1.94 & 0.45 & $48942.940 \ldots \ldots$ & 8.86 & 0.44 \\
\hline $46428.916 \ldots \ldots$ & 7.66 & 0.37 & $46890.799 \ldots \ldots$ & 4.53 & 0.24 & $50796.913 \ldots \ldots$ & -0.17 & 0.43 \\
\hline $46448.872 \ldots \ldots$ & 6.41 & 0.47 & $46896.672 \ldots \ldots$ & 3.64 & 0.29 & $50797.018 \ldots \ldots$ & 0.02 & 0.42 \\
\hline $46453.818 \ldots \ldots$ & 6.41 & 0.35 & $46918.597 \ldots \ldots$ & 5.31 & 0.30 & $50811.963 \ldots \ldots$ & -1.40 & 0.65 \\
\hline $46459.842 \ldots \ldots$ & 6.79 & 0.40 & $46931.628 \ldots \ldots$ & 5.54 & 0.39 & $50843.844 \ldots \ldots$ & -1.99 & 0.35 \\
\hline \multirow[t]{2}{*}{$46509.825 \ldots \ldots$} & 3.20 & 0.39 & $46952.549 \ldots \ldots$ & 7.01 & 0.37 & $50876.790 \ldots \ldots$ & -3.48 & 0.34 \\
\hline & & & BD +51 1817 & 130839.1 & +510359 & & & \\
\hline $46928.843 \ldots \ldots$ & -50.74 & 0.40 & $47339.723 \ldots \ldots$ & -56.85 & 0.60 & $48045.800 \ldots \ldots$ & -53.25 & 0.69 \\
\hline $47164.045 \ldots \ldots$ & -65.11 & 0.65 & $47368.596 \ldots \ldots$ & -54.97 & 0.86 & $49047.893 \ldots \ldots$ & -51.54 & 0.79 \\
\hline $47188.963 \ldots \ldots$ & -66.98 & 0.74 & $47518.860 \ldots \ldots$ & -53.45 & 1.51 & $49049.916 \ldots \ldots$ & -52.28 & 0.71 \\
\hline $47198.987 \ldots \ldots$ & -66.17 & 0.85 & $47524.871 \ldots$ & -53.53 & 0.91 & $49105.771 .$. & -56.60 & 0.72 \\
\hline $47199.970 \ldots \ldots$ & -67.18 & 0.65 & $47695.747 \ldots$ & -67.72 & 0.61 & $49107.750 \ldots \ldots$ & -56.61 & 0.84 \\
\hline $47200.956 \ldots \ldots$ & -66.72 & 0.64 & $47788.593 \ldots \ldots$ & -63.72 & 0.69 & $49109.745 \ldots \ldots$ & -56.26 & 0.66 \\
\hline $47227.888 \ldots \ldots$ & -65.99 & 0.73 & $47904.828 \ldots \ldots$ & -53.72 & 0.71 & $49116.759 \ldots \ldots$ & -55.98 & 0.67 \\
\hline $47252.875 \ldots \ldots$ & -64.16 & 0.73 & $47931.734 \ldots \ldots$ & -52.03 & 0.90 & $49122.708 \ldots \ldots$ & -57.98 & 0.71 \\
\hline $47283.882 \ldots \ldots$ & -60.96 & 0.62 & $47955.922 \ldots \ldots$ & -50.55 & 0.78 & & & \\
\hline \multirow[t]{2}{*}{$47306.738 \ldots \ldots$} & -59.08 & 0.70 & $48001.794 \ldots \ldots$ & -51.82 & 0.67 & & & \\
\hline & & & G66-30 & 145007.8 & +005027 & & & \\
\hline $46927.888 \ldots \ldots$ & -110.27 & 0.67 & & -111.22 & 0.91 & & -115.44 & 0.78 \\
\hline $47206.005 \ldots \ldots$ & -122.68 & 1.46 & $47695.767 .$. & -112.39 & 0.87 & 48785 & -114.61 & 1.00 \\
\hline $47214.896 \ldots \ldots$ & -121.75 & 1.16 & $47966.010 \ldots \ldots$ & -122.57 & 0.89 & $50884.948 \ldots \ldots$ & -112.20 & 0.96 \\
\hline $47220.905 \ldots \ldots$ & -121.43 & 1.14 & $47993.854 \ldots \ldots$ & -119.49 & 0.84 & $50914.893 \ldots \ldots$ & -112.59 & 0.83 \\
\hline $47231.770 \ldots \ldots$ & -123.76 & 1.48 & $48016.867 \ldots \ldots$ & -118.79 & 0.96 & $50948.771 \ldots \ldots$ & -111.08 & 0.84 \\
\hline $47308.697 \ldots \ldots$ & -118.93 & 0.75 & $48431.744 \ldots \ldots$ & -112.61 & 0.82 & $50998.685 \ldots \ldots$ & -110.90 & 1.05 \\
\hline $47578.037 \ldots \ldots$ & -111.06 & 1.05 & $48675.030 \ldots \ldots$ & -120.56 & 0.84 & $51234.028 \ldots \ldots$ & -114.72 & 0.77 \\
\hline $47603.925 \ldots \ldots$ & -111.43 & 0.82 & $48697.953 \ldots \ldots$ & -119.65 & 0.76 & $51267.908 \ldots \ldots$ & -117.33 & 0.90 \\
\hline \multirow[t]{2}{*}{$47630.850 \ldots \ldots$} & -110.10 & 0.94 & $48723.841 \ldots \ldots$ & -116.67 & 0.57 & $51293.825 \ldots \ldots$ & -119.09 & 0.78 \\
\hline & & & G202-5 & 163558.6 & +455159 & & & \\
\hline $45244.620 \ldots \ldots$ & -241.07 & 1.66 & $46251.728 .$. & -244.25 & 1.28 & $49078.913 \ldots \ldots$ & -231.34 & 0.86 \\
\hline $45475.859 \ldots \ldots$ & -256.03 & 0.58 & $46271.641 \ldots \ldots$ & -257.75 & 1.21 & $49079.952 \ldots \ldots$ & -233.41 & 0.94 \\
\hline $45567.606 \ldots \ldots$ & -235.98 & 1.08 & $46338.497 \ldots \ldots$ & -243.11 & 1.20 & $49080.961 \ldots \ldots$ & -234.95 & 1.09 \\
\hline $45570.569 \ldots \ldots$ & -237.90 & 1.39 & $46360.458 \ldots \ldots$ & -235.55 & 1.36 & $49081.995 \ldots \ldots$ & -232.95 & 1.04 \\
\hline $45623.471 \ldots \ldots$ & -258.43 & 3.68 & $46362.457 \ldots \ldots$ & -235.16 & 1.34 & $49082.963 \ldots \ldots$ & -233.64 & 0.90 \\
\hline $45771.998 \ldots \ldots$ & -260.96 & 0.62 & $46421.951 \ldots \ldots$ & -245.96 & 1.13 & $49106.850 \ldots \ldots$ & -249.36 & 1.05 \\
\hline $45774.024 \ldots \ldots$ & -260.04 & 0.68 & $46429.949 \ldots \ldots$ & -251.47 & 1.04 & $49107.913 \ldots \ldots$ & -249.42 & 1.01 \\
\hline $45833.801 \ldots \ldots$ & -244.74 & 0.83 & $46436.915 \ldots \ldots$ & -256.82 & 1.01 & $49108.930 \ldots \ldots$ & -249.79 & 0.99 \\
\hline $45863.864 \ldots \ldots$ & -232.54 & 1.04 & $46454.060 \ldots \ldots$ & -263.85 & 1.08 & $49109.921 \ldots \ldots$ & -251.88 & 1.03 \\
\hline $45896.639 \ldots \ldots$ & -230.99 & 1.74 & $46540.916 \ldots \ldots$ & -231.62 & 0.96 & $49110.800 \ldots \ldots$ & -253.46 & 1.11 \\
\hline $45949.557 \ldots \ldots$ & -264.25 & 1.58 & $49049.999 \ldots \ldots$ & -232.67 & 1.04 & $49111.842 \ldots \ldots$ & -252.93 & 0.89 \\
\hline $46135.818 \ldots \ldots$ & -259.55 & 1.24 & $49056.036 \ldots \ldots$ & -231.01 & 0.94 & $49112.840 \ldots \ldots$ & -253.25 & 1.06 \\
\hline $46193.822 \ldots \ldots$ & -236.71 & 1.05 & $49056.974 \ldots \ldots$ & -231.33 & 0.94 & $49113.851 \ldots \ldots$ & -254.81 & 0.94 \\
\hline $46216.804 \ldots \ldots$ & -232.09 & 1.19 & $49057.936 \ldots \ldots$ & -230.17 & 1.01 & $49114.883 \ldots \ldots$ & -255.17 & 0.96 \\
\hline $46223.770 \ldots \ldots$ & -231.85 & 1.00 & $49059.022 \ldots \ldots$ & -230.27 & 0.91 & $49115.894 \ldots \ldots$ & -256.06 & 0.83 \\
\hline $46224.783 \ldots \ldots$ & -229.42 & 1.54 & $49076.928 \ldots \ldots$ & -232.44 & 1.16 & & & \\
\hline $46246.595 \ldots \ldots$ & -241.13 & 1.06 & $49077.971 \ldots \ldots$ & -232.32 & 1.05 & & & \\
\hline
\end{tabular}


typical values of $v \sin i=0,1,2,4,6,8,10,12,16,20,25,30$, $35,40,50,60,70,80,90,100,120$, and $140 \mathrm{~km} \mathrm{~s}^{-1}$.

For each blue-straggler candidate we adopted the template with the effective temperature and metallicity nearest to the values for that star reported in Paper XII. We used $\log g=4.0$ throughout. We then ran grids of correlations to determine the rotational velocity, $v \sin i$, that gave the highest average value for the peak of the correlation function. The parameters adopted for the final correlations are coded in the final column of Table 1: " $t$ " specifies the effective temperature $(\mathrm{K})$, " $\mathrm{g}$ " indicates the $\log$ gravity $(\mathrm{cgs})$ times 10 , " $\mathrm{m}$ " shows the log of the metal abundance compared to solar (" $\mathrm{m}$ " for minus) times 10 , and " $\mathrm{v}$ " signifies the rotational velocity $\left(\mathrm{km} \mathrm{s}^{-1}\right)$. For all but one star the rotational velocities are small and represent only marginally significant detections. The exception is BD -122669 , with $v \sin i$ a bit faster than $30 \mathrm{~km} \mathrm{~s}^{-1}$, in excellent agreement with the value found by CP.

Table 2 contains the individual radial-velocity measurements, including the Heliocentric Julian Date of midexposure $(-2,400,000)$ and the velocity and internal error estimate returned by the IRAF task RVSAO, both in kilometers per second.

\section{RESULTS}

\subsection{Nonvariables}

Table 3 summarizes the results for four program stars that failed to display unambiguous variations in radial velocity despite coverage ranging from 15.9 to $19.0 \mathrm{yr}$. Columns (2) and (3) give the number of observations and the total observing span in days. Column (4) gives the mean radial velocity, and column (5) gives the preferred value of the rotational velocity, $v \sin i$, where the parentheses indicate a low level of confidence. Columns (6) and (7) give the standard deviation from the mean of the observed velocities and the average internal error estimate from RVSAO, and column (8) is the ratio of the two. The final column gives the probability that stars with constant velocity could have by accident the observed $\chi^{2}$ value or larger, assuming normally distributed errors. For many of our stars there is significant variation in the exposure level of the spectra for that star, so it is important to use the internal error estimate for each exposure, $\sigma_{i, \text { int }}$, when calculating $\chi^{2}$ :

$$
\chi^{2}=\sum_{i=1}^{n}\left(\frac{x_{i}-\left\langle x_{i}\right\rangle}{\sigma_{i, \text { int }}}\right)^{2} .
$$

Figure 2 of Paper XII showed that stars with $[\mathrm{m} / \mathrm{H}]$ ranging from -0.8 to -2.2 have single measure uncertainties of 0.7 to $1.1 \mathrm{~km} \mathrm{~s}^{-1}$, so the scatter for the four stars is consistent with little or no velocity variability. Plots of the velocities versus time and the power spectrum of the data also failed to reveal any obvious periodic velocity variations.
Jasniewicz \& Mayor (1988) obtained 11 radial-velocity measures for $\mathrm{BD}+251981$ over a period of 1862 days, finding a mean velocity of $+57.9 \mathrm{~km} \mathrm{~s}^{-1}$ and a standard error of $0.9 \mathrm{~km} \mathrm{~s}^{-1}$, consistent with our results.

While not obvious, one of these four stars also appears to be a spectroscopic binary with a very long period. Table 3 shows that the ratio of the external error $(\sigma$ of the measured values) to the internal error (estimated from the cross correlation software) is higher than expected for BD + 251981 (and to a lesser degree for HD 84937). We also derived $\chi^{2}$ values and computed the probability of the $\chi^{2}$ value, $P\left(\chi^{2}\right)$, which is given in column (8). The $P\left(\chi^{2}\right)$ value is also very low for BD + 251981 (and again to a lesser degree for HD 84937). However, these statistical indicators are vulnerable to observational errors that are not normally distributed, such as outliers. Perhaps more convincing is the hint of a gradual trend with time of the velocities for BD + 251981 , shown in Figure 2. We judge that the star's radial velocity has gone through a maximum some time around HJD $2,450,000$. There does not seem to be a long-term trend for HD 84937, and we assume that larger-than-average scatter during the early period of our observations has led to the large $E / I$ ratio and small $P\left(\chi^{2}\right)$ value. We believe that $\mathrm{BD}$ +251981 will prove to be a long-period spectroscopic binary, but long-term, dedicated observations will be required to test our speculation.

\subsection{Orbital Solutions}

CP commented on the apparent velocity variability of BD -12 2669. It required persistence for us to obtain good phase coverage to derive a reliable orbit, because the period is only slightly longer than $1 \mathrm{yr}$. Figure 3 reveals that our data cover the phases near minimum and maximum velocity, so the orbital amplitude and mass function are well constrained. The final orbital elements are given in Table 4. Note that the orbit is nearly circular.

HD 97916 is possibly an unusual star in its own right. Laird (1985) found the star to be a member of the relatively rare class of metal-poor dwarfs that have enhanced nitrogen abundances. (Laird 1985 found another such star, HD 166913, and confirmed the two cases discovered previously by Bessell \& Norris 1982 . Carbon et al. found an additional example.) We note, however, that Carbon et al. (1987) did not find HD 97916 to have significantly enhanced $[\mathrm{C} / \mathrm{Fe}]$ or $[\mathrm{N} / \mathrm{C}]$ ratios. With $[\mathrm{Fe} / \mathrm{H}]=-1.31$ the star only barely meets our color criterion for being a blue-straggler candidate. As noted in Table 1, HD 97916 shows line broadening, presumably due to axial rotation. Our metallicity extraction technique, employing synthetic spectra with lower rotational broadening, will underestimate the metallicity for rotating stars such as HD 97916. Chen et al. (2000) have undertaken detailed chemical abundance analyses of a large

TABLE 3

Stars With Little/No Detected Velocity Variation

\begin{tabular}{ccccccccc}
\hline \hline Star & $\begin{array}{c}N \\
(2)\end{array}$ & $\begin{array}{c}\text { Span } \\
(\text { days }) \\
(3)\end{array}$ & $\begin{array}{c}\left\langle V_{r}\right\rangle \\
\left(\mathrm{km} \mathrm{s}^{-1}\right) \\
(4)\end{array}$ & $\begin{array}{c}v \sin i \\
\left(\mathrm{~km} \mathrm{~s}^{-1}\right) \\
(5)\end{array}$ & $\begin{array}{c}\sigma\left[V_{r}(\mathrm{ext})\right] \\
\left(\mathrm{km} \mathrm{s}^{-1}\right) \\
(6)\end{array}$ & $\begin{array}{c}\sigma\left[V_{r}(\mathrm{int})\right] \\
\left(\mathrm{km} \mathrm{s}^{-1}\right) \\
(7)\end{array}$ & $\begin{array}{c}E / I \\
(8)\end{array}$ & $\begin{array}{c}P\left(\chi^{2}\right) \\
(9)\end{array}$ \\
\hline $\mathrm{BD}+7294 \ldots \ldots$. & 31 & 6341 & -268.6 & $(3)$ & 0.89 & 0.83 & 1.08 & 0.236 \\
$\mathrm{BD}+401166 \ldots \ldots$ & 27 & 6930 & +105.5 & 9 & 0.69 & 0.73 & 0.96 & 0.549 \\
$\mathrm{BD}+251981 \ldots \ldots$. & 66 & 5813 & +57.5 & 10 & 0.77 & 0.53 & 1.46 & 0.000002 \\
$\mathrm{HD} 84937 \ldots \ldots \ldots$. & 76 & 6944 & -15.3 & $(4)$ & 0.91 & 0.71 & 1.28 & 0.0021 \\
\hline
\end{tabular}



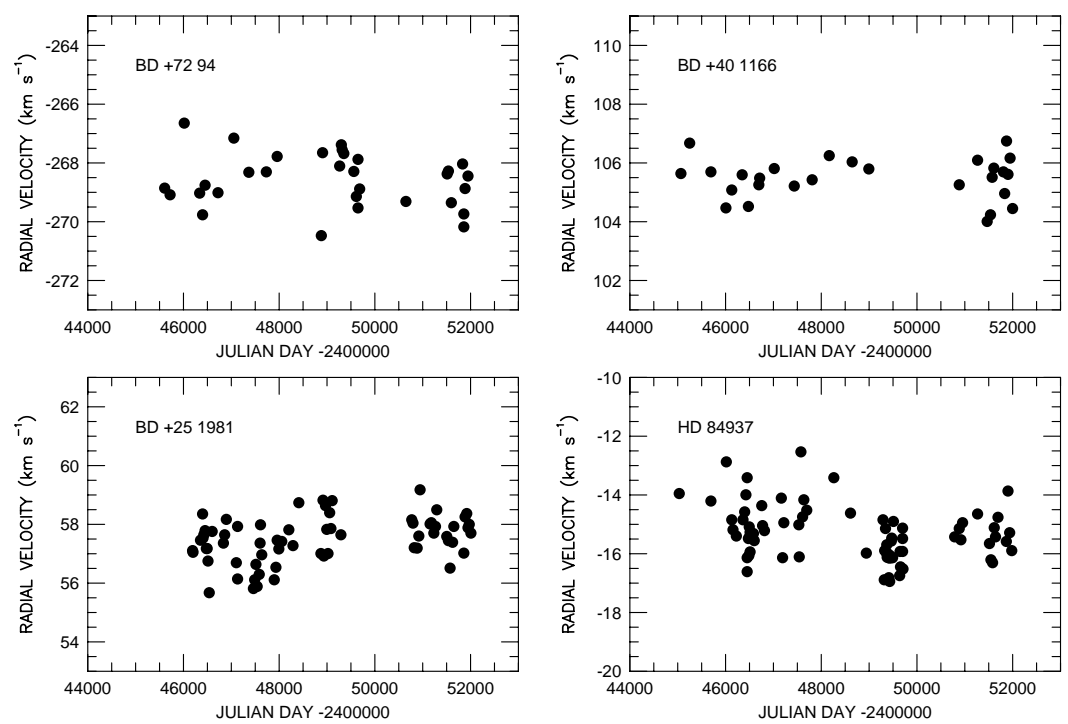

FIG. 2.-Histories of the measured radial velocities for BD + 72 94, BD +40 1166, BD + 25 1981, and HD 84937

number of field dwarfs, and they find $[\mathrm{Fe} / \mathrm{H}] \approx-1.00$ for HD 97916 (this is the average of the Fe I and Fe II results). In this case, $\Delta=-0.037$, and the star is well blueward of the nominal turnoff locus in Figure 1. HD 97916 turns out to be a binary star, with a nearly circular orbit.

Latham et al. (1992) presented an orbital solution for HD 106516 , based on radial velocities derived using an observed spectrum of the sky as a template. The orbital parameters listed in Table 4 are minor refinements, based on the use of a synthetic spectrum as the template and the inclusion of six new velocities. The scatter of the measured velocities about the orbital solution has decreased from 0.7 to $0.44 \mathrm{~km} \mathrm{~s}^{-1}$. The revised solution is shown in Figure 3. The orbit is very nearly circular. HD 106516 appears to show a modest rotational velocity, with $v \sin i \approx 10 \mathrm{~km} \mathrm{~s}^{-1}$. Photometric monitoring of its chromospheric activity indicates a rotational period of 6.9 days (Donahue, Saar, \& Baliunas 1996). With $[\mathrm{Fe} / \mathrm{H}]=-0.87$ and an enhancement of the " $\alpha$ " elements $(\mathrm{O}, \mathrm{Mg}, \mathrm{Si}, \mathrm{Ca}, \mathrm{Ti})$ averaging $[\alpha / \mathrm{Fe}] \approx+0.27$ (Edvardsson et al. 1993), the "effective" heavy element mass fraction $Z$ is
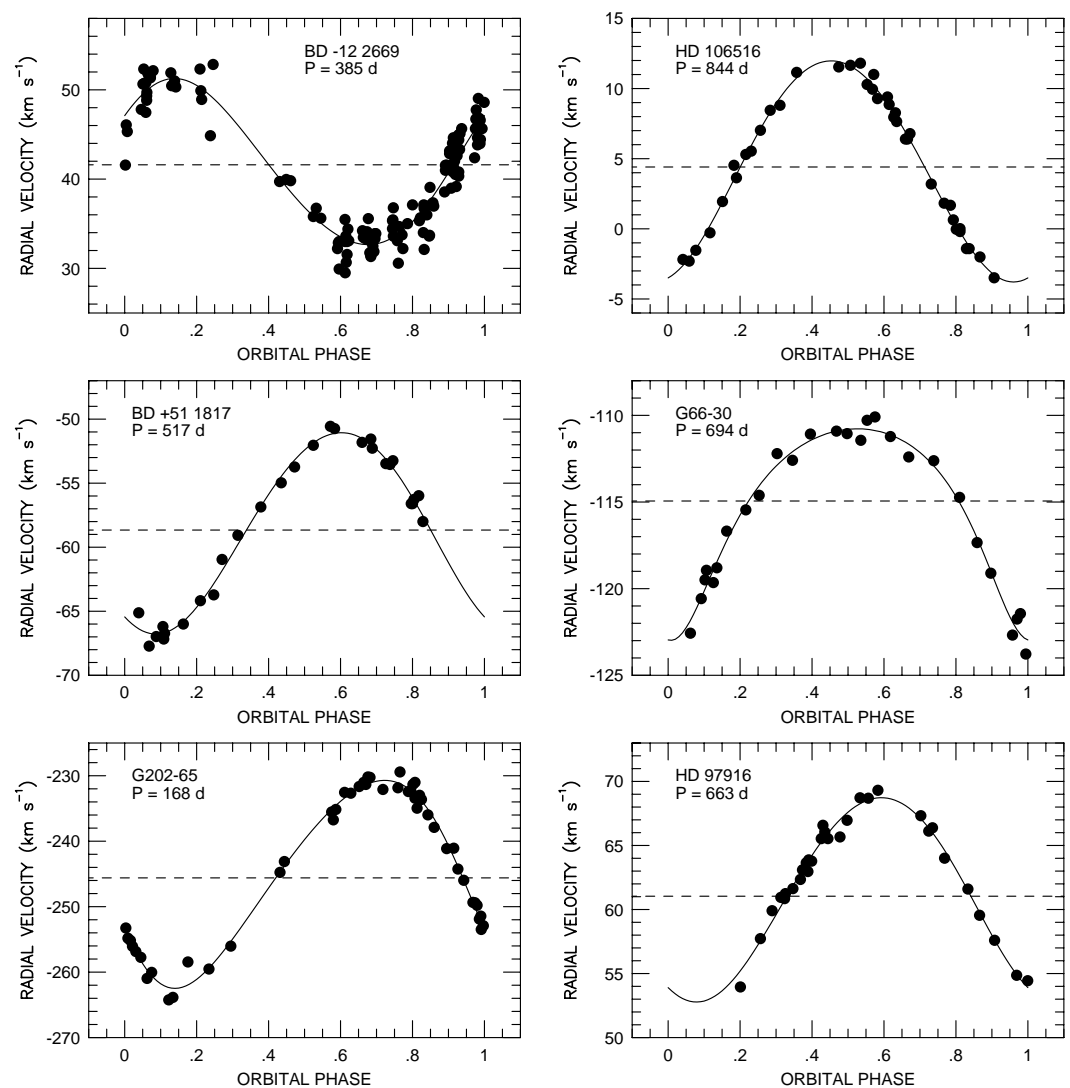

FIG. 3.-Orbital solutions and observed velocities for BD - 12 2669, HD 97916, HD 106516, BD + 51 1817, G66-30, and G202-65 
TABLE 4

Orbital Parameters

\begin{tabular}{|c|c|c|c|c|c|c|c|c|c|}
\hline Star & $\begin{array}{c}P \\
\text { (days) }\end{array}$ & $\begin{array}{c}\gamma \\
\left(\mathrm{km} \mathrm{s}^{-1}\right)\end{array}$ & $\begin{array}{c}K \\
\left(\mathrm{~km} \mathrm{~s}^{-1}\right)\end{array}$ & $e$ & $\begin{array}{c}\omega \\
(\mathrm{deg})\end{array}$ & $T_{0}$ & $\begin{array}{l}f(M) \\
\left(M_{\odot}\right)\end{array}$ & $N$ Span & $\begin{array}{r}\sigma(O-C) \\
\left(\mathrm{km} \mathrm{s}^{-1}\right)\end{array}$ \\
\hline \multirow{2}{*}{$\mathrm{BD}-122669 \ldots \ldots$} & 385.1 & +41.60 & 9.30 & 0.071 & 303.5 & 47257.2 & 0.0319 & 135 & 1.82 \\
\hline & \pm 1.0 & \pm 0.26 & \pm 0.28 & \pm 0.030 & \pm 25.0 & \pm 25.4 & \pm 0.0029 & 2097 & \\
\hline \multirow{2}{*}{ HD $97916 \ldots \ldots \ldots . .}$. & 663.36 & +61.04 & 7.98 & 0.042 & 149.3 & 46602.2 & 0.0348 & 32 & 0.61 \\
\hline & \pm 0.92 & \pm 0.16 & \pm 0.25 & \pm 0.024 & \pm 33.6 & \pm 62.7 & \pm 0.0032 & 5083 & \\
\hline \multirow[t]{2}{*}{ HD $106516 \ldots \ldots \ldots$} & $\overline{843.9}$ & +4.41 & 7.88 & 0.041 & 195.6 & $4 \overline{7580.3}$ & 0.0427 & 39 & 0.44 \\
\hline & \pm 1.1 & \pm 0.09 & \pm 0.13 & \pm 0.016 & \pm 20.2 & \pm 46.8 & \pm 0.0021 & 5181 & \\
\hline \multirow[t]{2}{*}{$\mathrm{BD}+511817 \ldots \ldots$} & $\overline{516.8}$ & -58.64 & 7.86 & 0.043 & 145.9 & $4 \overline{7660.5}$ & 0.0259 & 28 & 0.63 \\
\hline & \pm 2.2 & \pm 0.21 & \pm 0.18 & \pm 0.028 & \pm 43.0 & \pm 63.3 & \pm 0.0018 & 2194 & \\
\hline \multirow[t]{2}{*}{ G66-30........... } & 688.2 & -115.10 & 6.10 & 0.293 & 174.7 & 48611.9 & 0.0142 & 27 & 0.66 \\
\hline & \pm 2.0 & \pm 0.14 & \pm 0.19 & \pm 0.041 & \pm 7.1 & \pm 10.5 & \pm 0.0014 & 4366 & \\
\hline \multirow[t]{2}{*}{ G202-65 .......... } & $\overline{167.539}$ & $-\overline{245.60}$ & 15.89 & 0.145 & $\overline{115.5}$ & $4 \overline{7436.9}$ & 0.0674 & 49 & 1.10 \\
\hline & \pm 0.037 & \pm 0.26 & \pm 0.26 & \pm 0.016 & \pm 6.8 & \pm 2.9 & \pm 0.0033 & 3871 & \\
\hline \multirow[t]{2}{*}{ CS 22166-041 ...... } & $\overline{486.1}$ & +36.99 & 10.09 & 0.024 & 300.0 & 50226.0 & 0.0517 & 14 & 0.68 \\
\hline & \pm 1.9 & \pm 0.19 & \pm 0.25 & \pm 0.034 & \pm 84.0 & \pm 113.0 & \pm 0.0039 & 2203 & \\
\hline \multirow[t]{2}{*}{ CS $22170-028 \ldots \ldots$} & 0.976241 & -10.44 & 21.29 & 0.086 & 195.0 & 50274.631 & 0.000965 & 32 & 2.3 \\
\hline & \pm 0.000011 & \pm 0.53 & \pm 0.62 & \pm 0.034 & \pm 19.0 & \pm 0.052 & \pm 0.000084 & 1838 & \\
\hline \multirow[t]{2}{*}{ CS 22873-139 ...... } & 19.1611 & $+\overline{232} .20$ & 23.64 & 0.198 & 43.6 & $48 \overline{730} 0.95$ & 0.0247 & 47 & 3.3 \\
\hline & \pm 0.0056 & \pm 0.58 & \pm 0.69 & \pm 0.035 & \pm 8.6 & \pm 0.46 & \pm 0.0022 & 1461 & \\
\hline \multirow[t]{2}{*}{ CS 22876-008 ...... } & 302.99 & +67.28 & 8.80 & 0.075 & $\overline{131.0}$ & 50038.0 & 0.0212 & 13 & 0.82 \\
\hline & \pm 0.66 & \pm 0.24 & \pm 0.37 & \pm 0.044 & \pm 32 & \pm 26.0 & \pm 0.0027 & 2223 & \\
\hline \multirow[t]{2}{*}{ CS 22890-069 ..... } & 1.971796 & -2.23 & 33.6 & 0.053 & 270.0 & 50400.29 & 0.00771 & 53 & 7.2 \\
\hline & \pm 0.000061 & \pm 1.4 & \pm 1.3 & \pm 0.044 & \pm 60.0 & \pm 0.33 & \pm 0.00091 & 2193 & \\
\hline \multirow[t]{2}{*}{ CS $22892-027 \ldots \ldots$} & $\overline{485.2}$ & -22.03 & 5.73 & 0.259 & 84.0 & 50328.0 & 0.00850 & 31 & 0.61 \\
\hline & \pm 2.3 & \pm 0.15 & \pm 0.20 & \pm 0.041 & \pm 11.0 & \pm 17.0 & \pm 0.00090 & 2574 & \\
\hline \multirow[t]{2}{*}{ CS 22948-068 ...... } & 300.20 & +80.46 & 6.98 & 0.044 & 115.0 & 50191.0 & 0.0105 & 18 & 0.52 \\
\hline & \pm 0.54 & \pm 0.13 & \pm 0.18 & \pm 0.033 & \pm 32.0 & \pm 27.0 & \pm 0.0008 & 2224 & \\
\hline \multirow[t]{2}{*}{ CS $22956-028 \ldots \ldots$} & 1307.0 & +34.22 & 8.79 & 0.207 & 289.0 & 50211.0 & 0.086 & 21 & 1.09 \\
\hline & \pm 23.0 & \pm 0.32 & \pm 0.55 & \pm 0.055 & \pm 17.0 & \pm 66.0 & \pm 0.017 & 2198 & \\
\hline \multirow{2}{*}{ CS 22966-054 ...... } & 306.43 & -84.43 & 9.67 & 0.059 & 132.0 & 49994.0 & 0.0285 & 13 & 0.87 \\
\hline & \pm 0.81 & \pm 0.29 & \pm 0.43 & \pm 0.040 & \pm 37 & \pm 31.0 & \pm 0.0038 & 2207 & \\
\hline \multirow[t]{2}{*}{ S 29518-039 ....... } & 1577.0 & -37.63 & 7.69 & 0.075 & 94.0 & 49199.0 & 0.074 & 14 & 1.9 \\
\hline & \pm 72.0 & \pm 0.56 & \pm 0.70 & \pm 0.135 & \pm 74.0 & \pm 312.0 & \pm 0.020 & 2203 & \\
\hline
\end{tabular}

roughly 0.004 (Salaris, Chieffi, \& Straniero 1993). We used the $Y=0.23,10 \times 10^{9} \mathrm{yr}$ isochrone of Straniero \& Chieffi (1991) at $T_{\text {eff }} \approx 6100 \mathrm{~K}$ (Paper XII). The isochrone yields $M_{V}=4.39$, in excellent agreement with that obtained using the Hipparcos parallax $\left(M_{V}=4.35 \pm 0.05\right)$ and a radius of $1.09 R_{\odot}$. The resultant rotational velocity is about $7 \mathrm{~km}$ $\mathrm{s}^{-1}$, a bit lower than we estimate from the spectra.

Our second spectrum of BD +51 1817 revealed that it is a velocity variable, and Figure 3 shows our orbital solution. Again, the orbit is nearly circular.

Among our binary solutions, only G66-30 is substantially eccentric, with $e=0.29 \pm 0.04$, as shown in Figure 3 .

The velocity variability for G202-65 was also manifested with our second observation, and Figure 3 shows that the orbit has been well sampled since then. The eccentricity is somewhat higher than that of BD -122669 or BD +51 1817 , but as we discuss below, it is still small compared with the median eccentricity found for the main sample of longperiod, metal-poor field spectroscopic binaries.

\section{DISCUSSION}

\subsection{Binary Frequency}

For more than a decade we have been monitoring the radial velocities of 1414 stars in our proper-motion sample (Paper XII). Of these, 238 clearly have spectroscopic companions, and orbital solutions are now in hand for more than 200 . We find no significant difference in the frequency of binaries among the metal-poor and metal-rich stars (Latham et al. 2001), so we use the entire sample to estimate the frequency of spectroscopic binaries. Excluding our 10 field blue stragglers, the formal frequency of spectroscopic binary and multiple systems per target is $17.0 \pm 1.0 \%$, if we make no restrictions on the period range included nor corrections for selection effects or incompleteness. Therefore, this should not be taken as a definitive determination of the frequency of spectroscopic binaries. But it is useful for comparing with the frequency of binaries found among our field blue stragglers, which have been monitored in the same way and as part of the same sample. Six of the 10 field bluestraggler candidates have proved to be binary systems with orbital solutions. By this measure, the binary frequency, 60 $\pm 24 \%$ is nearly $2 \sigma$ higher than that for the normal mainsequence stars in the proper-motion sample, suggesting that the presence of a companion star is part of the cause of the field blue straggler phenomenon.

While the difference in the binary frequency of the blue stragglers and the remainder of the metal-poor stars in our survey is significant formally at less than the $2 \sigma$ level, there are three reasons why our blue-straggler binary frequency might be underestimated. First, our selection criterion may not have been $100 \%$ successful in eliminating normal stars near the turnoff locus. If a small fraction of the halo is younger than the rest, a few otherwise normal stars might be selected as blue-straggler candidates. In addition, photometric uncertainties in the $(B-V)_{0}$ values are at least 0.01 
mag, so normal stars very close to the turnoff locus could appear blueward of it by accident. Five stars, BD + 72 94, HD 84937, HD 97916, HD 106516, and G66-30, lie within $0.02 \mathrm{mag}$ of the turnoff locus, and thus some of them may have been included in Table 1 by observational accident. Excluding all five of these stars leaves the binary fraction among blue stragglers in our sample the same, $60 \%$, but with a larger uncertainty, $\pm 35 \%$. Second, we have noted that one of the stars in Table 3 may prove to be a longperiod spectroscopic binary (BD +25 1981). The five bluest stars would then have a binary frequency of $4 / 5=80$ $\pm 40 \%$. Finally, our sample may include one (or more) stars that originated in a small galaxy that was accreted by the Milky Way and whose metal-poor stars may be younger than those in our Galaxy (Preston et al. 1994). The relatively large velocity dispersion of the Preston et al. (1994) sample prevents us from identifying any specific star that entered our sample in this fashion, but, assuming the small galaxy's stars have a binary frequency like those in the Milky Way, the inclusion of such a star is more likely to lower the observed binary frequency than to increase it.

We argue, then, that our data are consistent with all field blue stragglers being binary systems. And there is additional evidence regarding the importance of binaries in the formation of blue stragglers.

After this paper was submitted for publication and refereed, we learned of the complementary work of Preston \& Sneden (2000). They followed up earlier work by Preston et al. (1994) on the blue metal-poor stars, which were selected from objective prism spectra and $U B V$ photometry using criteria similar to our own. That is, the stars had to appear to be metal-poor dwarfs bluer than a metal-poor globular cluster main-sequence turnoff, $B-V<0.35$. Preston et al. (1994) argued that most of their sample had belonged to a relatively young satellite galaxy that had been accreted by the Milky Way. Preston \& Sneden (2000) noted two especially interesting characteristics of the binaries in their sample. First, they reported that an unusually high fraction of the stars turned out to be spectroscopic binaries. They listed orbital solutions (or possible solutions) for 42 of the 62 stars in their sample, and for 35 of the 51 stars with $[\mathrm{Fe} / \mathrm{H}] \leq-0.6$ (the metallicity limit adopted for the present paper). The corresponding binary frequencies are $68 \pm 10 \%$ and $69 \pm 12 \%$, respectively. This is much higher than the frequency of binaries found for solar-type stars in the disk and halo populations, about $15 \%$ for periods shorter than 1000 days (see Duquennoy \& Mayor 1991; Latham et al. 2001). This high binary frequency suggests that either the cannibalized galaxy had a high binary fraction, which we find hard to accept, or that a binary-rich subsample has been included, such as blue stragglers. In fact, Preston \& Sneden (2000) used this result to revise upward their estimate of the density of Galactic field halo blue stragglers relative to young metal-poor stars from an accreted galaxy in their blue metal-poor sample.

The second interesting characteristic noted by Preston \& Sneden (2000) was an unusual distribution of orbital elements for the binaries in their sample, with a relatively large number of orbits with both low eccentricities and long periods, on the order of a few hundred days. Furthermore, they reported a tendency toward small secondary masses in their sample compared with the distributions reported by Duquennoy \& Mayor (1991) for G dwarfs in the solar neighborhood and by Latham et al. $(1988,1992)$ for metal- poor halo stars. Preston \& Sneden (2000) suggested that this might be due to the presence of a significant number of blue stragglers among their blue metal-poor stars and furthermore that the orbital characteristics provided a clue to the origin of the blue-straggler phenomenon.

We have reanalyzed the velocity data published by Preston \& Sneden (2000) to derive our own orbital solutions. According to the criteria that we normally use for identifying binaries (see Latham et al. 1992, 2001), 29 of the 62 stars are unambiguous binaries. This confirms the conclusion reported by Preston \& Sneden (2000), that the binary frequency is high. For 10 of the binaries with $[\mathrm{Fe} /$ $\mathrm{H}] \leq-0.6$ we were able to derive orbital solutions that we consider to be reliable. The orbital parameters for these solutions are listed in Table 4. The orbit for the doublelined binary CS 22873-139 is a single-lined solution based on the primary velocities reported by Preston (1994). Five of these have both low eccentricities and long periods, and they also have small secondary masses. This confirms the presence of a population with unusual orbital characteristics, which Preston \& Sneden (2000) identified as blue stragglers.

We explore in the following subsections some of the other evidence that supports field blue stragglers as the result of binary mass transfer, including their location in the $e$ versus $\log P$ diagram, evidence that the secondaries are white dwarfs, and evidence that lithium is depleted in their photospheres, which is a rare phenomenon for metal-poor stars hotter than about $5400 \mathrm{~K}$ (Thorburn 1994) but is consistent with mass transfer.

\subsection{Orbital Periods and Eccentricities}

In Figure 4 we plot the orbital eccentricities versus the logarithm of the period for the 160 single-lined spectroscopic binaries reported by Latham et al. (2001). Our six blue-straggler candidates from Table 4 are plotted as filled circles. The 10 Preston \& Sneden (2000) binaries from Table 4 are plotted as filled squares. Two significant points are revealed by the figure.

1. The orbital periods of our blue stragglers are long, 167 to 844 days, and up to about 1600 days for the Preston $\&$ Sneden (2000) blue stragglers (and even longer if BD +25 1981 proves to be a long-period spectroscopic binary).

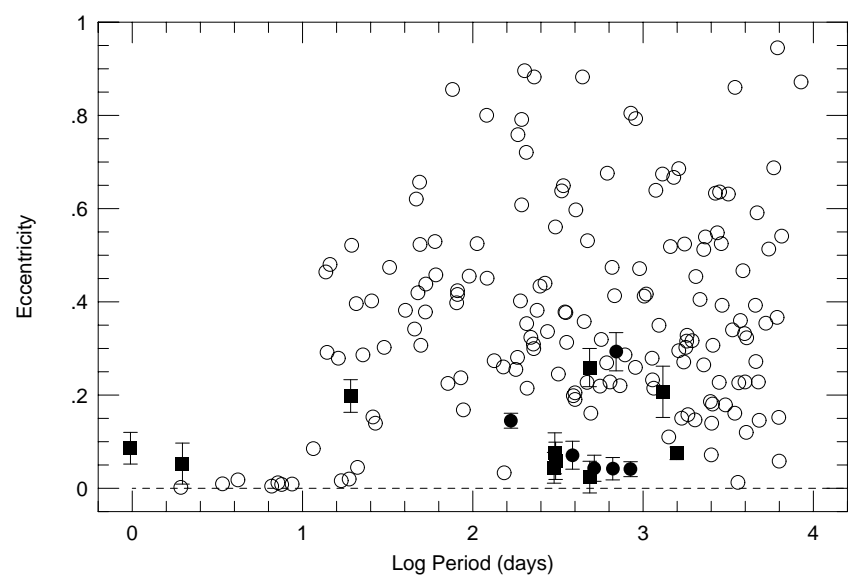

FIG. 4.-Orbital eccentricity vs. log period for 156 single-lined spectroscopic binaries from Latham et al. (2001) (open circles), the six bluestraggler binaries from this paper ( filled circles), and the 10 new orbits for blue metal-poor stars with $[\mathrm{Fe} / \mathrm{H}] \leq-0.6$ from Preston \& Sneden (2000) ( filled squares). 
2. Five of our six blue-straggler binaries have unusually low orbital eccentricities, with $e<0.15$ and $\langle e\rangle=0.068 \pm 0.020 \quad(\mathrm{rms}=0.044)$, compared with the median eccentricity of 0.37 for the 143 single-lined spectroscopic binaries with periods longer than 20 days (and thus not having been circularized by tidal forces). If we include the Preston \& Sneden (2000) binaries with periods longer than 20 days in Table 4,10 of the 13 orbits have $e<0.15$, or $\langle e\rangle=0.062 \pm 0.011(\mathrm{rms}=0.034)$. Both the mean eccentricity for all 13 binaries, $e=0.106 \pm 0.025(\mathrm{rms}=0.091)$, and the largest eccentricity, $e=0.29$, lie well below the median value of $e=0.37$ for normal main-sequence, singlelined spectroscopic binaries (see Duquennoy \& Mayor 1991; Latham et al. 2001).

The long periods imply fairly wide separations. Assuming a system mass of $1.5 M_{\odot}$, the separations range from 0.9 to 2.0 AU and are rather wide for mass transfer or any sort of interactions between two main-sequence stars. On the other hand, mass transfer involving a post-main-sequence star could result in such systems. A post-main-sequence star will develop a deep convection zone, and tidal interactions will become more effective, depending on a high power of the ratio of the stellar radius to stellar separation. Thus larger, cooler stars with deeper convective zones may circularize their orbits out to very long periods. In a paper in preparation on binary orbits among a sample of metal-poor field red giants, we find nearly circular orbits for periods approaching 500 days. The long periods of the blue stragglers would arise at a late stage in the binary mass transfer process. Initially, the larger mass post-main-sequence star transfers mass rapidly, and the orbit shrinks rapidly until the mass ratio approaches unity. Once the mass of the donor star is less than that of the main-sequence star, mass transfer proceeds more slowly, because the orbit widens as the mass ratio declines below unity and the period increases (for example, see Paczyński 1971). This scenario is consistent qualitatively with the long periods and low eccentricities that we observe. It also predicts that the unseen companions are now white dwarfs, which we discuss below. In this scenario we also expect there to be an upper limit to the orbital period of a blue-straggler binary. The limit is set by the maximum radius reached by the expanding red giant branch (RGB) or asymptotic giant branch (AGB) star. For long-enough orbital periods, with large-enough separations, stable Roche lobe overflow would not be possible. Paczyński (1971) outlined the basic physics. For an initial primary-to-secondary mass ratio of $M_{1} / M_{2}$, overflow will occur if

$$
\frac{R_{1}}{a}=0.38+0.2 \log \left(\frac{M_{1}}{M_{2}}\right)
$$

where $a$ is the orbital semimajor axis and $R_{1}$ is the radius of the primary. For the system,

$$
\log P=1.5 \log a-0.5 \log \left(M_{1}+M_{2}\right)-0.936,
$$

where $P$ is in days, $a$ is in units of solar radii, and the masses are in units of solar masses. Typical maximum sizes of metal-poor red giant branch stars are quite insensitive to the initial mass and are about $100 R_{\odot}$ (see Straniero \& Chieffi 1991). For a combined system mass of $1.5 M_{\odot}$ (see the discussion on secondary masses below) and $R_{1} \leq 100$ $R_{\odot}$, initial mass ratios of $1.5,2.0$, and 3.0 lead to upper limits to current orbital periods of about 350,320 , and 290 days, under the assumption that no mass is lost from the system. If mass is lost, the final orbital period is longer, and it is thus perhaps consistent with our longest period of 844 days. Other systems in which mass transfer almost certainly has occurred have even longer periods, exceeding 4000 days.

\subsection{Other Long-Period, Low-Eccentricity Spectroscopic Binaries}

There are other classes of spectroscopic binaries that are not in our survey but that share with our blue stragglers the properties of long periods and nearly circular orbits. These other classes most likely result from the transfer of mass from a core helium-burning star onto an initially lower mass secondary, since they show signs of helium burning (in the form of enhanced carbon abundances) and s-process enhancements. Mass transfer apparently continued until material enriched in the products of hydrogen and helium burning was deposited in the photosphere of the companion, and the initial primary was reduced to a white dwarf and appears now as the less massive star in the system.

The Ba II and CH stars are low-velocity-metal-rich and high-velocity-metal-poor giants, respectively. They are not quite luminous enough to be AGB stars, yet abundance enhancements in carbon and s-process abundances are often seen. Webbink (1986) was the first to consider the orbital elements of AGB stars as possible indicators of mass transfer. McClure \& Woodsworth (1990) summarized a decade-long study of the velocities of such stars, finding essentially all of them to be binaries. Further, like our field blue stragglers, the periods of the $\mathrm{CH}$ stars are long, 328 to 2954 days, and the eccentricities are low (indistinguishable from circular for five stars, and $e=0.09,0.10$, and 0.18 for the remaining three stars). The Ba II stars also show long periods, 70 to 4400 days, and their orbital eccentricities are somewhat larger than those of their cousins (only eight of the 16 have $e<0.1$ ), but still low compared with binary systems containing normal $\mathrm{G}$ and $\mathrm{K}$ giants. The situation for the $\mathrm{Ba}$ II stars is probably more complex than a single mode of mass transfer, however, as described by Han et al. (1995). Multiple channels for their formation appear to be possible, including wind accretion, wind exposure, stable Roche-lobe overflow, and common envelope ejection. Their models suggest that the stable Roche-lobe overflow may have been more common in systems with orbital periods now in the range of 100 to 2000 days. Udry et al. (1998a, 1998b) have extended the work of McClure \& Woodsworth (1990), obtaining orbital solutions for 26 strong barium stars. Of the 16 such stars with periods less than 2000 days, and adding in HD 121447 (Jorissen et al. 1995), the orbital eccentricities vary from 0.23 down to values indistinguishable from circular. The mean eccentricity is $0.074 \pm 0.018$ $(\sigma=0.072)$, considerably smaller than the typical eccentricity of 0.23 for normal giant binaries (Boffin, Paulus, \& Cerf 1992). Thus "case C" mass transfer (from an AGB star via stable Roche-lobe overflow) is a plausible explanation for most of these shorter period strong barium stars, and for the $\mathrm{CH}$ stars as well. Ultraviolet detections of the expected white dwarf companions have been obtained by BöhmVitense (1980), Dominy \& Lambert (1983), Böhm-Vitense \& Johnson (1985), and Böhm-Vitense et al. (2000).

The subgiant $\mathrm{CH}$ stars discovered by Bond (1974) are thought to be the progenitors of moderately metal-deficient Ba II stars (Smith, Coleman, \& Lambert 1993). As McClure 
(1989) has noted, such stars have also been called "dwarf barium stars," and they overlap with the "F Str $\lambda 4077$ " stars (North \& Duquennoy 1991). McClure (1989) found that at least seven of the 10 such stars under study are indeed binaries, while North \& Duquennoy (1992) also reported a high frequency of binaries. Luck \& Bond (1991) proposed the explicit connection between blue stragglers and the $\mathrm{CH}$ stars. Further, they reported orbital solutions for three stars, and all show relatively long periods (73, 1208 , and 1316 days) and small eccentricities $(0.18,0.17$, 0.04). McClure (1997) has recently summarized the results of his 15 years of radial-velocity monitoring of subgiant $\mathrm{CH}$ stars. Nine of the 10 stars are binaries, and he reported orbital solutions for six of them. Like the $\mathrm{Ba}$ II and $\mathrm{CH}$ stars, the periods of the subgiant $\mathrm{CH}$ stars are long: 878 to 4140 days; and the eccentricities are small, ranging from $0.09 \pm 0.08$ to $0.16 \pm 0.08$.

Finally, the "dwarf carbon stars" are another, but rarer, class of probable "case C" mass transfer (de Kool \& Green 1995). Their rarity is probably a selection effect resulting from two factors: lower luminosity and higher temperature. The dwarf luminosities are low compared with the Ba II and $\mathrm{CH}$ stars, so the volume of space reached in magnitudelimited surveys is smaller for the dwarf carbon stars. The cooler temperatures of the $\mathrm{Ba}$ II and $\mathrm{CH}$ stars also makes spectral identification of the carbon enhancements more readily visible through the molecular features. An orbital solution has been obtained for the first member of the class to be discovered, G77-61. Like the blue stragglers, it has a moderately long period (245 days) and a nearly circular orbit (Dearborn et al. 1986).

In summary, all of the special classes of binaries discussed above appear to involve mass transfer from a core heliumburning star, leading to binary orbits with long periods and low eccentricities. Thus, the long periods and low eccentricities of our six blue stragglers support the idea that mass transfer has been involved. However, it is not clear whether the transfer began when the initial primary star was a firstascent red giant (inert helium core, hydrogen-burning shell) or an AGB star (inert $\mathrm{C}-\mathrm{O}$ core, helium-burning and hydrogen-burning shells).

\subsection{Mass Functions}

If the long periods and low eccentricities of our bluestraggler binaries have resulted from mass transfer, what should remain of the original primary that donated its mass? be

The mass function for a spectroscopic binary is defined to

$$
f(M)=\sin ^{3} i \frac{M_{2}^{3}}{\left(M_{1}+M_{2}\right)^{2}},
$$

where the numerical value of $f(M)$ is derived from the parameters of the orbital solution. McClure \& Woodsworth (1990) used their extensive orbital data for the Ba II and CH stars to compare the mass functions of both classes with those of normal $\mathrm{G}$ and $\mathrm{K}$ giants. They found that the Ba II and $\mathrm{CH}$ binaries show a preferred range for the secondary masses, consistent with those of normal field white dwarfs, $M_{2} \approx 0.55 M_{\odot}$. McClure (1997) found a very similar distribution of mass functions for the subgiant $\mathrm{CH}$ stars, so the secondaries of these stars apparently also have masses typical of white dwarfs.

Although we have derived fewer orbits for our field blue stragglers than in the case of the $\mathrm{CH}$ stars, we can carry out a similar analysis. We prefer to use a more suggestive form of the expression for the mass function, which we rewrite as

$$
M_{2} \sin i=f^{1 / 3}(M) \times\left(M_{1}+M_{2}\right)^{2 / 3} .
$$

If we can make a reliable estimate for the mass of the star that we now see, $M_{1}$, then we can use this equation to calculate the minimum mass of the undetected secondary, i.e., for $\sin i=1$. We have used the isochrones from Girardi et al. (2000) to estimate values of $M_{1}$. These isochrones cover a good range in metallicity and have helium abundances scaled with metallicity. To accommodate high temperatures and stars likely to be more luminous than zero-age main-sequence stars, we employ their $2.0 \mathrm{Gyr}$ isochrones. We assume $[\alpha / \mathrm{Fe}]=+0.3$ and use the $[\mathrm{Fe} / \mathrm{H}]$ values to calculate $Z_{\text {eff }}$ for each primary. We then use the temperatures to read off each primary mass, interpolating between isochrones as needed. The results of this analysis are summarized in Table 5.

The minimum masses for the secondaries are all smaller than the canonical value for the mass of a white dwarf, $\sim 0.55 M_{\odot}$. This is consistent with all the secondaries being white dwarfs in orbits viewed at inclinations less than $90^{\circ}$. If we assume that all the secondaries have the same mass of $0.55 M_{\odot}$ and calculate the orbital inclinations that this

TABLE 5

\begin{tabular}{|c|c|c|c|c|c|c|c|c|}
\hline Star & $e$ & {$[\mathrm{Fe} / \mathrm{H}]$} & $(B-V)_{0}$ & $T_{\text {eff }}$ & $Z_{\text {eff }}$ & $M_{1}$ & $\begin{array}{c}M_{2} \\
(\mathrm{~min})\end{array}$ & $\sin i$ \\
\hline BD -12 2669 . & 0.07 & -1.49 & 0.28 & 6710 & 0.0010 & 0.95 & 0.384 & 0.756 \\
\hline HD $97916 \ldots \ldots \ldots$ & 0.04 & -1.00 & 0.41 & 6235 & 0.0031 & 0.92 & 0.391 & 0.767 \\
\hline HD $106516 \ldots \ldots \ldots$ & 0.04 & -0.87 & 0.45 & 6100 & 0.0042 & 0.92 & 0.426 & 0.822 \\
\hline $\mathrm{BD}+511817 \ldots \ldots$ & 0.04 & -1.10 & 0.38 & 6340 & 0.0025 & 0.92 & 0.346 & 0.695 \\
\hline G66-30 ........... & 0.29 & -1.75 & 0.38 & 6310 & 0.0017 & 0.83 & 0.256 & 0.546 \\
\hline G202-65 .......... & 0.14 & -1.50 & 0.36 & 6340 & 0.0010 & 0.87 & 0.503 & 0.935 \\
\hline CS 22166-041 ...... & 0.02 & -1.32 & 0.32 & 6560 & 0.0015 & 0.94 & 0.468 & 0.884 \\
\hline CS 22876-008 ...... & 0.07 & -1.37 & 0.28 & 6630 & 0.0013 & 0.95 & 0.326 & 0.659 \\
\hline CS 22892-027 ...... & 0.26 & -1.03 & 0.30 & 6720 & 0.0029 & 1.04 & 0.241 & 0.505 \\
\hline CS 22948-068 ...... & 0.04 & -1.88 & 0.31 & 6590 & 0.0004 & 0.89 & 0.237 & 0.508 \\
\hline CS 22956-028 ...... & 0.21 & -2.08 & 0.34 & 6335 & 0.0003 & 0.84 & 0.550 & 1.000 \\
\hline CS 22966-054 ...... & 0.06 & -1.17 & 0.28 & 6785 & 0.0021 & 1.01 & 0.381 & 0.747 \\
\hline CS 29518-039 ...... & 0.07 & -2.49 & 0.30 & 6510 & 0.0001 & 0.87 & 0.523 & 0.964 \\
\hline
\end{tabular}

Primary Mass Estimates AND $\sin i$ Assuming $M_{2}=0.55 M_{\odot}$ 
would imply, we get the $\sin i$ values listed in Table 5 . The average $\sin i$ for our six blue-straggler binaries is $\langle\sin i\rangle=0.75 \pm 0.05(\mathrm{rms}=0.13)$, close to the value of $\pi /$ $4=0.785$ expected for randomly oriented orbits. If we restrict this calculation to the five binaries with $e<0.15$, $\langle\sin i\rangle=0.80 \pm 0.04(\mathrm{rms}=0.09)$. The average $\sin i$ for the seven Preston \& Sneden (2000) binaries reported in Table 4 with periods longer than 20 days is $\langle\sin i\rangle=0.75 \pm 0.08$ $(\mathrm{rms}=0.21)$. If we restrict the calculation to the five binaries with $e<0.15,\langle\sin i\rangle=0.75 \pm 0.07(\mathrm{rms}=0.18)$. It appears that the two surveys produce similar values, so we combine them to find that all 13 binaries in Table 5 have $\langle\sin i\rangle=0.75 \pm 0.05(\mathrm{rms}=0.17)$.

We conclude that our orbital solutions are consistent with all the unseen companions in our low-eccentricity long-period orbits being white dwarfs viewed at random orbital orientations, which supports the mass-transfer model for the formation of field blue stragglers. If all the secondaries are white dwarfs with the same mass, then that mass cannot be much smaller than $0.50 M_{\odot}$, because the three largest minimum masses are $0.55 \pm 0.06 M_{\odot}$ (CS 22956-028), $0.52 \pm 0.06 M_{\odot}$ (CS 29518-039), and $0.50 \pm 0.01 M_{\odot}(\mathrm{G} 202-65)$, where the quoted errors include only the contribution from the mass functions and not from the primary masses. (However, we discuss below that CS 22956-028 may be a slightly different case than the other stars.) On the other hand, the secondary masses cannot be larger than about $0.65 M_{\odot}$, because then the average value for the orbital inclinations would become inconsistent with random orientations of the orbits. For example, if we assume that the mass of all 13 secondaries increases from $M_{2}=0.55$ to $0.65 M_{\odot},\langle\sin i\rangle$ decreases from $0.75 \pm 0.05$ to $0.67 \pm 0.04$. Likewise, for the 10 stars with $e \leq 0.15$, the decrease in $\langle\sin i\rangle$ is from $0.77 \pm 0.04$ to $0.68 \pm 0.04$.

The fact that the secondary masses for our blue-straggler binaries are similar to those of the binary systems found in $\mathrm{Ba}$ II stars, $\mathrm{CH}$ stars, and subgiant $\mathrm{CH}$ stars, and that all of these are similar to the masses of normal field white dwarfs, suggests that they all had comparably long periods of time to grow the core mass to the same degree.

The fact that all our blue-straggler binaries are singlelined implies that we have not detected any light from the secondaries, which is consistent with the secondaries all being white dwarfs.

\subsection{Blue Straggler Abundances}

Photospheric chemical abundances may also address the question of whether mass transfer has occurred and, if so, in what evolutionary state the original primary was when the transfer began.

\subsubsection{Lithium}

The relatively fragile element lithium is expected to be depleted in the case of mass transfer (and also in the case of internal mixing in a star that has not experienced mass transfer, if the mixing reaches all the way to the photosphere).

Surface abundances of lithium decline with effective temperature for $T_{\text {eff }}<5400 \mathrm{~K}$ among metal-poor dwarfs (see Thorburn 1994 and references therein) and in subgiants (Pilachowski, Sneden, \& Booth 1993), presumably due to the increasing depth of the envelope convective zone. However, among the hotter main-sequence dwarfs and subgiants, lithium abundances in metal-poor stars show a typical value of $\log n(\mathrm{Li})=2.1$ (Pilachowski et al. 1993; Ryan et al. 2001b). What are the lithium abundances in our program stars? Are they truly indicators in favor of mass transfer?

We begin with the six binary stars with orbital solutions. The rotational broadening of BD -122669 has inhibited searches for its $\lambda 6707 \mathrm{Li}$ I feature. G202-65 is deficient in lithium (Hobbs \& Mathieu 1991; Ryan et al. 2001a), but we must point out that this star has a temperature comparable to the "lithium gap" first seen in the study of Hyades dwarfs by Boesgaard \& Budge (1988). The gap is probably not related to mass transfer, so it is not clear that the lithium deficiency seen in G202-65 must be interpreted as evidence for mass transfer, but merely that it is at least consistent with mass transfer. The other four stars are all cooler than the gap and are all deficient in lithium. HD 97916 has been known to be highly deficient in lithium for almost two decades (Spite, Maillard, \& Spite 1984), with $\log n(\mathrm{Li})<1.2$. HD 106516 has $\log n(\mathrm{Li}) \leq 1.3$ (Spite, Pasquini, \& Spite 1994; Lambert, Heath, \& Edvardsson 1991; Boesgaard \& Tripicco 1986). Further, it is also very deficient in beryllium (Molaro et al. 1997), with $\log n(\mathrm{Be})<-0.76$. BD +511817 has $\log n(\mathrm{Li})<-1.6$ (Ryan et al. 2001a), and G66-30 has $\log n(\mathrm{Li})<1.5$ (Spite et al. 1993; Ryan et al. 2001a). Thus all five of our field bluestraggler candidates with orbital solutions and measured lithium abundances are deficient in lithium, which is consistent with mass transfer (although the lithium deficiency for G202-65 could have been caused by or enhanced by whatever phenomenon causes the lithium gap).

Let us now consider the two blue-straggler candidates that are much hotter than the main-sequence turnoff and also hotter than the lithium gap. No lithium abundance analyses have been done for BD +40 1166, but BD +25 1981 has been found to be deficient in lithium, with $\log n(\mathrm{Li}) \leq$ 1.2 (Glaspey et al. 1994). (And we remind the reader that the latter star does appear to be a long-period binary.)

We turn now to the remaining two stars that are only slightly (if at all) hotter than the main-sequence turnoff, BD + 7294 and HD 84937. The lithium abundances in both stars are normal (BD + 72 94, Pilachowski et al. 1993; Ryan et al. 1996; HD 84937, Ryan et al. 1999; Ryan et al. 1996; Thorburn 1994).

If we argue that their normal lithium abundances disqualify these two blue-straggler candidates, we note that the binary frequency of our field blue stragglers rises to six out of eight, or $75 \pm 31 \%$. If we add BD +251981 , the binary fraction rises to seven out of eight $(88 \pm 33 \%)$. And BD +40 1166 certainly needs to have its $[\mathrm{Fe} / \mathrm{H}]$ value confirmed and its lithium abundance measured. It is possible that all field blue stragglers are characterized by long-period, low-eccentricity binary systems with the primary star deficient in lithium and the secondary star being a normal-mass white dwarf.

There are a few other interesting stars not included in our study. Hobbs \& Mathieu (1991) and Pritchet \& Glaspey (1991) have found lithium deficiencies in blue stragglers both inside and outside the gap in the old open cluster M67. Glaspey et al. (1994) attempted to measure lithium abundances in five other metal-poor, high-velocity blue stragglers. While most of these stars lie in the lithium gap, BD +2374 is hotter than the gap and was found to be deficient in lithium. This star in particular deserves an extensive radial-velocity study (which is now underway at the CfA). 
The deficiencies of lithium (and presumably beryllium) also link the blue stragglers to the subgiant $\mathrm{CH}$ stars as well as the $\mathrm{Ba}$ II and $\mathrm{CH}$ stars, all of which are highly deficient in lithium (Lambert, Smith, \& Heath 1993; Smith et al. 1993). Note that prior work suggesting higher lithium abundances in the Ba II stars (Pinsonneault, Sneden, \& Smith 1984; Barbuy et al. 1992) has been superseded by the work of Lambert et al. (1993), who found that the supposed lithium feature in such stars is due to an unidentified s-process element, probably cerium. There is one interesting exception to the lithium deficiencies: CS 22898-27, with a spectroscopic gravity typical of subgiants and enhanced $s$-process abundances, which led McWilliam et al. (1995) to suggest that it is a subgiant $\mathrm{CH}$ star. However, Thorburn (1994) has found its lithium abundances to be enhanced. Radial-velocity monitoring of this star is recommended. We note that lithium enhancements have been seen in a few highly evolved metal-poor stars, including the Population II Cepheid M5-V42 (Carney, Fry, \& Gonzalez 1999), the red giant IV-101 in M3 (Kraft et al. 1999), and the very luminous and cool variable star V2 in NGC 362 (Smith, Shetrone, \& Keane 1999). The rarity of this phenomenon suggests that if mixing can provide enhanced photospheric lithium abundances, the result is short lived.

It appears that in all the studied examples of long-period, nearly circular binary systems discussed above, lithium is deficient (although we note that the $\mathrm{Ba}$ II and $\mathrm{CH}$ stars may also be sufficiently evolved to have destroyed the lithium during their otherwise normal evolution).

\subsubsection{AGB Signatures: Carbon and s-Process Elements}

We have noted that the orbital characteristics of most of the field blue-straggler binaries appear similar to those of the $\mathrm{Ba} \mathrm{II}, \mathrm{CH}$, subgiant $\mathrm{CH}$, and dwarf carbon stars, which are thought to result from mass transfer from core heliumburning stars. It therefore is worth exploring the abundances of carbon and the s-process elements in the blue stragglers. Unfortunately, little is known about such stars' elemental abundances. CP studied BD +25 1981 and BD -122669 , and they concluded that $[\mathrm{Ba} / \mathrm{Fe}]$ is roughly solar in the former. However, this is the expected value for stars with $[\mathrm{Fe} / \mathrm{H}] \approx-1.3$ (Gratton \& Sneden 1994; François 1996), and, unfortunately, the uncertainty in $[\mathrm{Ba} / \mathrm{Fe}]$ is very large, \pm 0.5 dex.

HD 106516 has been studied extensively, and the similarities with the carbon and barium stars fail. $[\mathrm{C} / \mathrm{Fe}]$ is normal (Tomkin et al. 1995), as are the s-process elements yttrium, zirconium, and barium (Edvardsson et al. 1993). This suggests that, at least in the case of this star, the blue straggler is not a result of the same evolutionary process that leads to barium or $\mathrm{CH}$ stars. The mass transfer and orbital evolution may have been similar, but the masslosing star may have been in a different evolutionary state or the mass transfer may have been less complete so that the abundance anomalies are not as dramatic as in the Ba II, $\mathrm{CH}$, subgiant $\mathrm{CH}$, or dwarf carbon stars. Could the masslosing star have been a red giant rather than an AGB star? We note that among the metal-poor globular clusters, some of the more evolved red giants show oxygen deficiencies and sodium enhancements (see reviews by Kraft 1994 and Carney 1996). Edvardsson et al. (1993) found normal abundances of both oxygen and sodium in HD 106516, which does not support the red giant hypothesis. Unfortunately, the photospheric evidence for mixing is a complicated subject insofar as Kraft et al. (1992) and Pilachowski, Sneden, \& Kraft (1996), in their comparisons of abundances in metal-poor field and cluster red giants, have suggested that field giants appear to be less well mixed than cluster giants.

Preston \& Sneden (2000) derived chemical abundances for a large number of their blue metal-poor stars, including the light $s$-process element strontium and the heavy $s$ process element barium. They noted that the blue-straggler candidates in their sample, defined as those stars with periods longer than 25 days and low orbital eccentricities, did not show any signs of enhanced s-process abundances. The number of strontium and barium lines available for study was small, and we have attempted a simple average of these abundances for all the longperiod binaries $(P>25$ days) within a limited metallicity range. We concentrated on metal-poor stars $([\mathrm{Fe} / \mathrm{H}] \leq-0.6)$ but avoided stars that are extremely metal-poor $([\mathrm{Fe} / \mathrm{H}] \leq-2.0)$, because Galactic nucleosynthesis involving only a few individual supernovae at such an early stage may lead to unusual elemental abundance ratios, as noted by Preston \& Sneden (2000) and in the extensive work by Burris et al. (2000). We also chose to include only the stars that are relatively narrow lined $\left(v \sin i \leq 25 \mathrm{~km} \mathrm{~s}^{-1}\right)$ and have excluded the pulsating SX Phe variables. The five stars satisfying these criteria, excluding CS 22956-028, have $\langle[\mathrm{Sr} / \mathrm{Fe}]\rangle=+0.12 \pm 0.09$ and $\langle[\mathrm{Ba} / \mathrm{Fe}]\rangle=+0.18 \pm 0.08$. The errors are those of the mean. As in the case of HD 106516, the blue-straggler candidates of Preston \& Sneden (2000) $(e \leq 0.15)$ do not have distinguishably different abundances of $s$-process elements than normal metal-poor stars (Burris et al. 2000). For CS 22956-028, with the longest period of the 13 stars and $e \approx 0.21$, the strontium and barium appear to be enhanced, $[\mathrm{Sr} / \mathrm{Fe}]=+1.14$, and $[\mathrm{Ba} / \mathrm{Fe}]=+0.42$ (Preston \& Sneden 2000). This may reflect the increased scatter found by Burris et al. (2000) for stars with $[\mathrm{Fe} / \mathrm{H}] \leq-2$. On the other hand, this may be a hint that in this case the mass donor was an AGB star, which might explain both the longperiod and the unusual abundances. If true, this star's evolution may produce a subgiant $\mathrm{CH}$ star, as suggested by Luck \& Bond (1991).

We urge more detailed studies of the chemical abundances, especially lithium and $s$-process elements, in the other long-period, low-eccentricity blue straggler binaries.

\section{SUMMARY AND FUTURE WORK}

We have argued that existing observations are consistent with the model that all field blue stragglers are binary systems with long periods and low orbital eccentricities and with primary stars that are deficient in lithium and secondary stars that are normal-mass white dwarfs. All these points are consistent with mass transfer as the cause of the blue straggler phenomenon. The fraction of spectroscopic binaries among our candidate metal-poor field blue stragglers (six out of 10) is high and is consistent with all field blue stragglers being binaries. These stars are distinguished from the normal metal-poor spectroscopic binaries by the prevalence of orbits with long periods and low eccentricities. They share these characteristics with stars that are almost certainly formed by mass transfer from evolved stars (core helium burning in these cases): the $\mathrm{Ba}$ II and $\mathrm{CH}$ stars, the subgiant $\mathrm{CH}$ stars, and the dwarf carbon stars. Further, the blue-straggler binaries show marked lithium deficiencies, which may also be explained (readily) by mass 
transfer. And finally, it appears that the secondaries in the binary blue stragglers have masses consistent with white dwarfs, as has been found for the Ba II and CH stars (McClure \& Woodsworth 1990) and for the subgiant CH stars (McClure 1997). The subgiant CH stars and the Ba II and $\mathrm{CH}$ stars also show lithium deficiencies, but we note that the blue stragglers have not been shown to be a direct link to such stars since for the stars studied no anomalous carbon or $s$-process abundances were seen.

We recommend further studies of several issues that might help explain the blue-straggler phenomenon in lowdensity environments. First, do the metal-poor, highvelocity, field blue stragglers identified by Stetson (1991) and found to be lithium deficient by Glaspey et al. (1994) show signs of binary companions? If so, what are their orbital characteristics? (We note that we have been observing four more such stars and have found all of them to be spectroscopic binaries. We are continuing our observations to derive the orbital parameters.) The blue metal-poor Preston \& Sneden binaries should also be included in this work. Second, are there any signs of the products of red giant or AGB nucleosynthesis in the photospheres of blue stragglers that would help reveal more about when the mass transfer began? We strongly recommend detailed abundance analyses, including carbon, oxygen, sodium, magnesium, aluminum, and s-process elements, of all the metal-poor field blue stragglers, particularly those with loweccentricity binary orbits. BD +40 1166 especially needs a high-resolution, high-S/N abundance analysis to confirm its $[\mathrm{Fe} / \mathrm{H}]$ value and measure its lithium abundance and hence its candidacy as a field blue straggler.

On the theoretical side, it would be interesting to see whether detailed models that include the effects of stellar evolution, mass transfer, and orbital evolution can produce the characteristics observed for these blue-straggler binaries.
Finally, we remind the reader that our blue stragglers have been identified using the colors of globular cluster main-sequence turnoffs as a guide. However, mass transfer from an evolved star to a main-sequence star need not result in a star that is more massive and hence bluer and brighter than the turnoff for single main-sequence stars, if the original secondary has a low-enough mass. It may therefore be worthwhile to look in more detail at the other binaries in Figure 4 that have long periods but low eccentricities. Further, Ryan et al. (2001a) have called attention to stars that appear to be main-sequence dwarfs, which are cooler than the turnoff temperature and very deficient in photospheric lithium. They speculated that mass transfer, such as we have discussed for the field blue stragglers, may have been involved. Radial-velocity studies are certainly needed for these stars, and such work is underway at the CfA.

We thank Tsevi Mazeh for his many contributions to the tools that we use to solve the orbits of spectroscopic binaries and Guillermo Torres for his implementation of those tools at CfA. We also are very grateful to Bob Kurucz for his help through the years with his model atmosphere and synthetic spectrum codes. As always, we are indebted to the many people who made observations for this project, especially Robert Stefanik, Jim Peters, Ed Horine, Alejandra Milone, and Bob Davis. It is a pleasure to thank the National Science Foundation for financial support to the University of North Carolina and to Bowling Green State University during the many years that we have been puzzling over the blue-straggler phenomenon. Finally, we thank the referee for his/her constructive and unusually careful review.

\section{REFERENCES}

Bailyn, C. D. 1995, ARA\&A, 33, 133

Barbuy, B., Jorissen, A., Rossi, S. C. F., \& Arnould, M. 1992, A\&A, 262, 216

Bessell, M. S., \& Norris, J. 1982, ApJ, 263, L29

Boesgaard, A. M., \& Budge, K. G. 1988, ApJ, 332, 410

Boesgaard, A. M., \& Tripicco, M. J. 1986, ApJ, 303, 724

Boffin, H. M. J., Paulus, G., \& Cerf, N. 1992, in Binaries as Tracers of Stellar Formation, ed. A. Duquennoy \& M. Mayor (Cambridge: Cambridge Univ. Press), 26

Böhm-Vitense, E. 1980, ApJ, 239, L79

Böhm-Vitense, E., Carpenter, K., Robinson, R., Ake, T., \& Brown, J. 2000, ApJ, 533, 969

Böhm-Vitense, E., \& Johnson, H. R. 1985, ApJ, 293, 288

Bond, H. E. 1974, ApJ, 194, 95

Bond, H. E., \& MacConnell, D. J. 1971, ApJ, 165, 51

Brown, J. A., Wallerstein, G., \& Zucker, D. 1996, in ASP Conf. Ser. 92,

Formation of the Galactic Halo-Inside and Out, ed. H. Morrison \& A. Sarajedini (San Francisco: ASP), 355

Buonanno, R., Corsi, C. E., Fusi Pecci, F., Richer, H. B., \& Fahlman, G. G. 1993, AJ, 105, 184

Burris, D. L., Pilachowski, C. A., Armandroff, T. E., Sneden, C., Cowan, J. J., \& Roe, H. 2000, ApJ, 544, 302

Burstein, D., \& Heiles, C. 1982, AJ, 87, 1165

Carbon, D. F., Barbuy, B., Kraft, R. P., Friel, E. D., \& Suntzeff, N. B. 1987, PASP, 99,335

Carney, B. W. 1996, PASP, 108, 900

Carney, B. W., Fry, A. M., \& Gonzalez, G. 1998, AJ, 116, 2984

Carney, B. W., Laird, J. B., Latham, D. W., \& Aguilar, L. A. 1996, AJ, 112, 668

Carney, B. W., Laird, J. B., Latham, D. W., \& Kurucz, R. L. 1987, AJ, 94, 1066

Carney, B. W., Latham, D. W., \& Laird, J. B. 1989, AJ, 97, 423

Carney, B. W., Latham, D. W., Laird, J. B., \& Aguilar, L. A. 1994, AJ, 107, 2240 (Paper XII)

Carney, B. W., \& Peterson, R. C. 1981, ApJ, 251, 190 (CP)
Chen, Y. Q., Nissen, P. E., Zhao, G., Zhang, H. W., \& Benoni, T. 2000, A\&AS, 141, 491

Dearborn, D. S. P., Liebert, J., Aaronson, M., Dahn, C. C., Harrington, R., Mould, J., \& Greenstein, J. L. 1986, ApJ, 300, 314

de Kool, M., \& Green, P. J. 1995, ApJ, 449, 236

Dominy, J. F., \& Lambert, D. L. 1983, ApJ, 270, 180

Donahue, R. A., Saar, S. H., \& Baliunas, S. L. 1996, ApJ, 466, 384

Duquennoy, A., \& Mayor, M. 1991, A\&A, 248, 485

Edvardsson, B., Andersen, J., Gustafsson, B., Lambert, D. L., Nissen, P. E., \& Tomkin, J. 1993, A\&A, 275, 101

Ferraro, F. R., Fusi Pecci, F., \& Bellazzini, M. 1995, A\&A, 294, 80

Ferraro, F. R., Fusi Pecci, F., Cacciari, C., Corsi, C. E., Buonanno, R., Fahlman, G. G., \& Richer, H. B. 1993, AJ, 106, 2324

François, P. 1996, A\&A, 313, 229

Girardi, L., Bressan, A., Bertelli, G., \& Chiosi, C. 2000, A\&AS, 141, 371

Glaspey, J. W., Pritchet, C. J., \& Stetson, P. B. 1994, AJ, 108, 271

Gratton, R. G., \& Sneden, C. 1994, A\&A, 287, 927

Han, Z., Eggleton, P. P., Podsiadlowski, P., \& Tout, C. A. 1995, MNRAS, 277,1443

Hobbs, L. M., \& Mathieu, R. D. 1991, PASP, 103, 431

Jasniewicz, G., \& Mayor, M. 1988, A\&A, 203, 329

Jorissen, A., Hennen, O., Mayor, M., Bruch, A., \& Sterken, C. 1995, A\&A, 301, 707

Kraft, R. P. 1994, PASP, 106, 553

Kraft, R. P., Peterson, R. C., Guhathakurta, P., Sneden, C., Fulbright, J. P., \& Langer, G. E. 1999, ApJ, 518, L53

Kraft, R. P., Sneden, C., Langer, G. E., \& Prosser, C. F. 1992, AJ, 104, 645

Kurtz, M. J., \& Mink, D. J. 1998, PASP, 110, 934

Kurucz, R. L., Furenlid, I., Brault, J., \& Testerman, L. 1984, Solar Flux Atlas from 296 to $1300 \mathrm{~nm}$, (Sunspot: National Solar Obs.)

Laird, J. B. 1985, ApJ, 289, 556

Laird, J. B., Carney, B. W., \& Latham, D. W. 1988, AJ, 95, 1843

Lambert, D. L., Heath, J. E., \& Edvardsson, B. 1991, MNRAS, 253, 610

Lambert, D. L., Smith, V. V., \& Heath, J. 1993, PASP, 105, 568 
Latham, D. W. 1985, in IAU Coll. No. 88, Stellar Radial Velocities, ed. A. G. D. Philip \& D. W. Latham (Schenectady: L. Davis Press), 21 1992, in IAU Coll. No. 135, Complementary Approaches to Binary and Multiple Star Research, ed. H. McAlister \& W. Hartkopf (ASP Conf. Ser. 32) (San Francisco: ASP), 110

Latham, D. W., Mazeh, T., Carney, B. W., McCrosky, R. E., Stefanik, R. P., \& Davis, R. J. 1988, AJ, 96, 567

Latham, D. W., Mazeh, T., Stefanik, R. P., Davis, R. J., Carney, B. W., Krymolowski, Y., Laird, J. B., Torres, G., \& Morse, J. A. 1992, AJ, 104, 774

Latham, D. W., Stefanik, R. P., Torres, G., Davis, R. J., Mazeh, T., Carney, B. W., Laird, J. B., \& Morse, J. A. 2001, AJ, submitted

Leonard, P. J. T. 1996, in ASP Conf. Ser. 90, The Origins, Evolution, and Destinies of Binary Stars in Clusters, ed. E. F. Milone \& J.-C. Mermilliod (San Francisco: ASP), 337

Livio, M. 1993, in ASP Conf. Ser. 53, Blue Stragglers, ed. R. E. Saffer (San Francisco: ASP), 3

Luck, R. E., \& Bond, H. E. 1991, ApJS, 77, 515

Mathys, G. 1991, A\&A, 245, 467

McClure, R. D. 1989, in IAU Coll. No. 106, Evolution of Peculiar Red Giant Stars, ed. H. R. Johnson \& B. Zuckerman (Cambridge: Cambridge Univ. Press), 196 1997, PASP, 109, 536

McClure, R. D., \& Woodsworth, A. W. 1990, ApJ, 352, 709

McCrea, W. H. 1964, MNRAS, 128, 147

McWilliam, A., Preston, G. W., Sneden, C., \& Searle, L. 1995, AJ, 109, 2757

Molaro, P., Bonifacio, P., Castelli, F., \& Pasquini, L. 1997, A\&A, 319, 593

Morse, J. A., \& Kurucz, R. L. 2001, in preparation

Nemec, J. M., \& Cohen, J. G. 1989, ApJ, 336, 780

Nemec, J. M., Mateo, M., Burke, M., \& Olszewski, E. W. 1995, AJ, 110, 1186

Nemec, J. M., Nemec, A. F. L., \& Lutz, T. E. 1994, AJ, 108, 222

Nordström, B., Latham, D. W., Morse, J. A., Milone, A. A. E., Kurucz, R. L., Andersen, J., \& Stefanik, R. P. 1994, A\&A, 287, 338

North, P., \& Duquennoy, A. 1991, A\&A, 244, 335

. 1992, in Binaries as Tracers of Stellar Formation, ed A. Duquennoy \& M. Mayor (Cambridge: Cambridge Univ. Press), 202 Paczyński, B. 1971, ARA\&A, 9, 183

Pilachowski, C. A., Sneden, C., \& Booth, J. 1993, ApJ, 407, 699

Pilachowski, C. A., Sneden, C., \& Kraft, R. P. 1996, AJ, 111, 1689

Pinsonneault, M. H., Sneden, C., \& Smith, V. V. 1984, PASP, 96, 239
Preston, G. W. 1994, AJ, 108, 2267

Preston, G. W., Beers, T. C., \& Shectman, S. A. 1994, AJ, 108, 538

Preston, G. W., \& Landolt, A. U. 1998, AJ, 115, 2515

Preston, G. W., \& Sneden, C. 2000, AJ, 120, 1014

Pritchet, C. J., \& Glaspey, J. W. 1991, ApJ, 373, 105

Ryan, S. G., Beers, T. C., Deliyannis, C. P., \& Thorburn, J. A. 1996, ApJ, 458,543

Ryan, S. G., Beers, T. C., Kajino, T., \& Rosolankova, K. 2001a, ApJ, 547, 231

Ryan, S. G., Norris, J. E., \& Beers, T. C. 1999, ApJ, 523, 654

Ryan, S. G., Kajino, T., Beers, T. C., Suzuki, T. K., Romano, D., Matteucci, F., \& Rosolonkova, K. 2001b, ApJ, 549, 55

Saio, H., \& Wheeler, J. C. 1980, ApJ, 242, 1176

Salaris, M., Chieffi, A., \& Straniero, O. 1993, ApJ, 414, 580

Sandage, A. R. 1953, AJ, 58, 61

Schuster, W. J. \& Nissen, P. E. 1988, A\&AS, 73, 225

. 1989, A\&A, 221, 65

Schuster, W. J., Parrao, L., \& Contreras Martínez, M. E. 1993, A\&AS, 97, 951

Shara, M. M., Saffer, R. A., \& Livio, M. 1997, ApJ, 489, L59

Smith, V. V., Coleman, H., \& Lambert, D. L. 1993, ApJ, 417, 287

Smith, V. V., Shetrone, M. D., \& Keane, M. J. 1999, ApJ, 516, L73

Spite, M., Maillard, J. P., \& Spite, F. 1984, A\&A, 141, 56

Spite, M., Molaro, P., François, P., \& Spite, F. 1993, A\&A, 271, L1

Spite, M., Pasquini, L., \& Spite, F. 1994, A\&A, 290, 217

Stetson, P. B. 1991, AJ, 102, 589

Straniero, O., \& Chieffi, A. 1991, ApJS, 76, 525

Stryker, L. L. 1993, PASP, 105, 1081

Thorburn, J. A. 1994, ApJ, 421, 318

Tomkin, J., Woolf, V. M., Lambert, D. L., \& Lemke, M. 1995, AJ, 109, 2204

Trimble, V. 1993, in ASP Conf. Ser. 53, Blue Stragglers, ed. R. Saffer (San Francisco: ASP), 155

Udry, S., Jorissen, A., Mayor, M., \& Van Eck, S. 1998a, A\&AS, 131, 25

Udry, S., Mayor, M., Van Eck, S., Jorissen, A., Prévot, L., Grenier, S., \& Lindgren, H. 1998b, A\&AS, 131, 43

Webbink, R. F. 1986, in Critical Observations versus Physical Models for Close Binary Systems, ed. K.-C. Leung \& D. S. Zhai (New York: Gordon \& Breach), 403

Wheeler, J. C. 1979a, ApJ, 234, 569

.1979b, Commun. Astrophys., 8, 133

Willson, L. A., Bowen, G. H., \& Struck-Marcell, C. 1987, Commun. Astrophys. 12,17 